Article

\title{
Cavitation on Model- and Full-Scale Marine Propellers: Steady and Transient Viscous Flow Simulations at Different Reynolds Numbers
}

\author{
Ville Viitanen $^{1, *}$, Timo Siikonen ${ }^{2} \mathbb{D}$ and Antonio Sánchez-Caja ${ }^{1}$ \\ 1 VTT Technical Research Centre of Finland Ltd, 02150 Espoo, Finland; Antonio.Sanchez@vtt.fi \\ 2 Department of Mechanical Engineering, Aalto University, 02150 Espoo, Finland; timo.siikonen@aalto.fi \\ * Correspondence: ville.viitanen@vtt.fi
}

Received: 9 January 2020; Accepted: 5 February 2020; Published: 20 February 2020

check for updates

\begin{abstract}
In this paper, we conducted numerical simulations to investigate single and two-phase flows around marine propellers in open-water conditions at different Reynolds number regimes. The simulations were carried out using a homogeneous compressible two-phase flow model with RANS and hybrid RANS/LES turbulence modeling approaches. Transition was accounted for in the model-scale simulations by employing an LCTM transition model. In model scale, also an anisotropic RANS model was utilized. We investigated two types of marine propellers: a conventional and a tip-loaded one. We compared the results of the simulations to experimental results in terms of global propeller performance and cavitation observations. The propeller cavitation, near-blade flow phenomena, and propeller wake flow characteristics were investigated in model- and full-scale conditions. A grid and time step sensitivity studies were carried out with respect to the propeller performance and cavitation characteristics. The model-scale propeller performance and the cavitation patterns were captured well with the numerical simulations, with little difference between the utilized turbulence models. The global propeller performance and the cavitation patterns were similar between the model- and full-scale simulations. A tendency of increased cavitation extent was observed as the Reynolds number increases. At the same time, greater dissipation of the cavitating tip vortex was noted in the full-scale conditions.
\end{abstract}

Keywords: marine propeller; CFD; cavitation simulation; turbulence modeling; scale effects

\section{Introduction}

Marine propeller cavitation can appear in a range of different forms, depending on the operating conditions of the propeller. Typical cavitation types encountered include steady attached sheet cavitation on the blade surface, transient bubble or cloud cavitation on the blade or in the wake, and vortex cavitation at the propeller tip and hub. The propeller cavitation can cause many detrimental effects, and the various types of cavitation usually result in different consequences from the points of view of the propeller material (e.g., erosion), the passengers (e.g., discomfort), or the ship's mission (e.g., identification and detectability). This further amplifies the need of accurate cavitation prognoses via experimental and numerical methodologies.

The propeller performance characteristics can notably vary in non-cavitating conditions at different Reynolds number regimes [1,2]. These aspects have been addressed before [2-6]: for instance, Rijpkema et al. [1] rigorously analyzed two different marine propellers using viscous computational fluid dynamics (CFD), which operated at different Reynolds numbers in non-cavitating conditions. Baltazar et al. [5,6] examined the $\gamma-R e_{\theta}$ transition model for a wetted propeller performance prediction. Sánchez-Caja et al. [2] investigated the scale effects of a CLT propeller experimentally and 
numerically. Figure 1 shows an example of paint tests on rough and smooth surfaces representative of full- and model-scale flow regimes. In laminar flow zones, the paint streaks on the blade surface tend to deviate from a circumferential path towards a radial direction (smooth surface) due to the lower shear stress. Conversely, the paths in the streaks corresponding to the rough surface are purely circumferential, which is indicative of turbulent flow. Typically, a distinct curve can be drawn, which separates these two flow zones, as in Figure 1. The location of transition from laminar to turbulent flow, and the extent of the different flow zones, are quite sensitive to the Reynolds number regime in which the propeller operates.
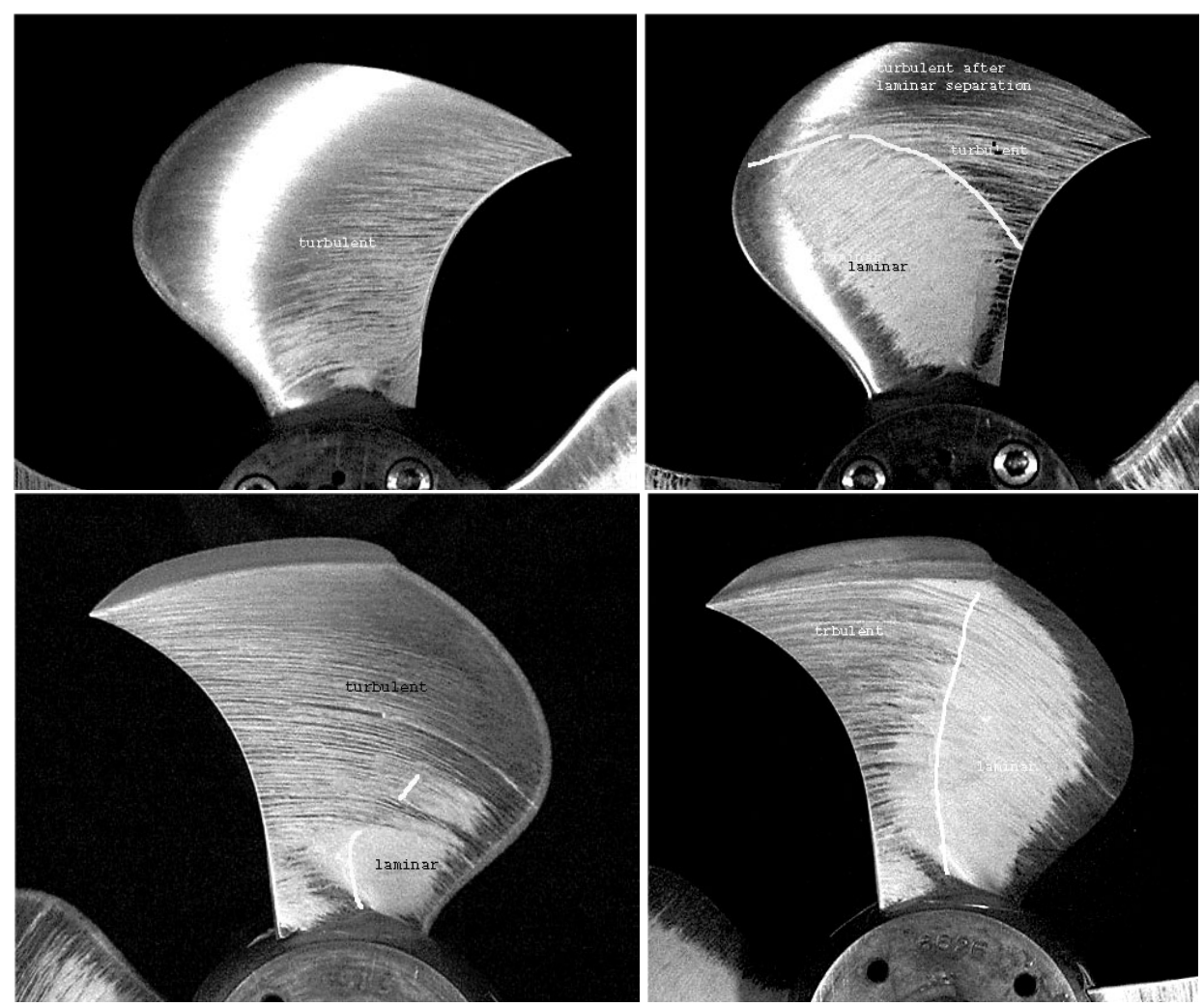

Figure 1. An example of paint test results for a CLT propeller [2]. The left frames show rough surfaces and the right frames smooth surfaces. Reproduced with permission from Juan Gonzalez-Adalid (Sistemar).

Reynolds number effects on the performance of a cavitating propeller or on the various forms of propeller cavitation structures are, however, less often reported [7,8]. The aim of this paper is to investigate numerically the scale effects on propeller cavitation, both in cases that feature steady and unsteady cavitation. Our goal is to gain deeper insight into the differences that may be found when predicting full-scale cavitation performance based on model test results. In the case of conventional, non-depressurized cavitation tanks, the differences also result from the impossibility to attain the cavitation number identity simultaneously at all radial stations. However, conventional facilities can use higher RPMs, guaranteeing larger Reynolds numbers, i.e., turbulent flow conditions over the blades. This may avoid the use of artificial leading edge roughness usually utilized in depressurized tanks due to the low rotational speed that may be required for cavitation scaling. In this study, we simulate viscous cavitating flows in model scale as they occur in conventional tunnels.

We investigate the model- and full-scale propellers in a uniform inflow condition. The propeller performance is predicted numerically utilizing viscous CFD. The range of Reynolds numbers studied covers those usually applied in the propeller open-water and cavitation tunnel model test $\left(\operatorname{Re}=\mathcal{O}\left(10^{5} \ldots 10^{6}\right)\right)$ to typical full-scale values $\left(\operatorname{Re}=\mathcal{O}\left(10^{7} \ldots 10^{8}\right)\right)$. We investigate two types of marine propellers: a conventional one and a tip-loaded one. Model-scale conditions are studied with four different turbulence modeling approaches: the shear stress transport (SST), both with and 
without transition modeling; an anisotropic Reynolds stress model; and with delayed-detached eddy simulation (DDES), which is a hybrid RANS/LES model (Reynolds-averaged Navier-Stokes/large eddy simulation). Full-scale conditions are studied with the SST turbulence model and with DDES.

This paper is organized as follows. Section 2 details the utilized viscous flow solver FINFLO, and Section 3 describes the test cases. In Section 4, we validate our numerical approach and assess the sensitivity of the model- and full-scale results to the used grid and time steps. We investigate the cavitation at different Reynolds number regimes in Section 5, and the near-blade flow including skin-friction and pressure distributions in Section 6. Finally, conclusions are drawn in Section 7.

\section{Flow Solution}

\subsection{Governing Equations}

The flow model applied is based on a homogeneous mixture flow assumption, and a compressible form of the Navier-Stokes equations is solved $[9,10]$. The continuity and momentum equations are solved for the mixture, and the energy equations are included to predict the correct acoustic signal speeds. The governing equations are

$$
\begin{aligned}
\frac{\partial \alpha_{k} \rho_{k}}{\partial t}+\nabla \cdot \alpha_{k} \rho_{k} \mathbf{V} & =\Gamma_{k} \\
\frac{\partial \rho \mathbf{V}}{\partial t}+\nabla \cdot \rho \mathbf{V} \mathbf{V}+\nabla p & =\nabla \cdot \boldsymbol{\tau}_{i j}+\rho \mathbf{g}
\end{aligned}
$$

where $p$ is the pressure, $\mathbf{V}$ the absolute velocity in a global non-rotating coordinate system, and $\boldsymbol{\tau}_{i j}$ the stress tensor, $\alpha_{k}$ a void (volume) fraction of phase $k, \rho_{k}$ the density, $t$ the time, $\Gamma_{k}$ the mass-transfer term, and $\mathbf{g}$ the gravity vector. The void fraction is defined as $\alpha_{k}=\mathscr{V}_{k} / \mathscr{V}$, where $\mathscr{V}_{k}$ denotes the volume occupied by phase $k$ of the total volume, $\mathscr{V}$. For the mass transfer, $\sum_{k} \Gamma_{k}=0$ holds, and consequently only a single mass-transfer term is needed.

Although the phase temperatures do not play a significant role in cavitation, the energy equations are always solved in the present method. The aim is to apply a compressible form of the equations. To predict the correct acoustic signal speeds, a complete model is needed. The phase temperatures $T_{g}$ and $T_{l}$ also have some influence on the solution via the material properties that are calculated as functions of the pressure and phase temperatures. The calculation of the material properties is described in [11]. The sound speed $c$ for a two-phase mixture is defined as

$$
\frac{1}{\rho c^{2}}=\frac{\alpha}{\rho_{g} c_{g}^{2}}+\frac{1-\alpha}{\rho_{l} c_{l}^{2}} \text { and } \frac{1}{c_{k}^{2}}=\frac{\partial \rho_{k}}{\partial p}+\frac{1}{\rho_{k}} \frac{\partial \rho_{k}}{\partial h_{k}}
$$

In the expressions above, the indices $g$ and $l$ refer to gas and liquid phases, respectively, and $h_{k}$ denotes the enthalpy of phase $k$.

The energy equations for phase $k=g$ or $l$ are written as

$$
\begin{array}{r}
\frac{\partial \alpha_{k} \rho_{k}\left(e_{k}+\frac{V^{2}}{2}\right)}{\partial t}+\nabla \cdot \alpha_{k} \rho_{k}\left(e_{k}+\frac{V^{2}}{2}\right) \mathbf{V}= \\
-\nabla \cdot \alpha_{k} \mathbf{q}_{k}+\nabla \cdot \alpha_{k} \boldsymbol{\tau}_{i j} \cdot \mathbf{V}+q_{i k}+\Gamma_{k}\left(h_{k s a t}+\frac{V^{2}}{2}\right)+\alpha_{k} \rho_{k} \mathbf{g} \cdot \mathbf{V}
\end{array}
$$

Here, $e_{k}$ is the specific internal energy, $\mathbf{q}_{k}$ the heat flux, $q_{i k}$ interfacial heat transfer from the interface to phase $k$, and $h_{k s a t}$ saturation enthalpy. Since $\sum_{k} \Gamma_{k}=0$, by adding the energy equations together, the following relationship is obtained between the interfacial heat and mass transfer,

$$
\Gamma_{g}=-\frac{q_{i g}+q_{i l}}{h_{g s a t}-h_{l s a t}} \quad \text { and } \quad q_{i k}=h_{i k}^{\prime}\left(T_{\text {sat }}-T_{k}\right)
$$


Above, $h_{i k}^{\prime}$ is a heat transfer coefficient between the phase $k$ and the interface. The interfacial heat transfer coefficients are based on the mass transfer, as shown in Section 2.3.

As compared to the single-phase flow, the momentum and total continuity equations in the homogeneous model do not change, except for the material properties like density and viscosity, which are calculated as

$$
\rho=\sum_{k} \alpha_{k} \rho_{k} \quad \text { and } \quad \mu=\sum_{k} \alpha_{k} \mu_{k}
$$

where $\mu$ is the dynamic viscosity. The turbulence effects are currently handled using single-phase models for the mixture.

The finite volume method is utilized to discretize the governing equations. The viscous fluxes as well as the pressure terms are centrally differenced. For the convective part, the variables on the cell surfaces are evaluated using a third-order upwind-biased monotonic upstream-centered scheme for conservation laws (MUSCL) interpolation. A compressive flux limiter has been shown to improve the predicted tip vortex cavitation pattern [10], and such is applied for the void fraction in the present study. For the time derivatives, a second-order three-level fully implicit method is used.

\subsection{Turbulence Modelling}

Nominally, a Reynolds-averaged form of the Navier-Stokes equations is used, and the DDES approach [12] that combines RANS and LES is also applied in the same form. In the present study, the calculations are performed up to the wall both in the model- and full-scale simulations, avoiding the use of wall functions. The height of the first cell was adjusted such that the non-dimensional wall distance $y^{+}=\rho u_{\tau} y / \mu \lesssim 1$ for the first cell, with $u_{\tau}=\sqrt{\tau_{w} / \rho}$ being the friction velocity, $\tau_{w}$ the wall shear stress, and $y$ is the normal distance from the solid surface to the center point of the cell next to the surface.

The base turbulence closure applied in the present calculations is the SST $k-\omega$ model [13]. That is a zonal model, referring to the formulation where the $k-\omega$ equations are solved only inside the boundary layer, and the standard $k-\varepsilon$ equations, transformed to the $\omega$-formulation, are solved away from the walls. The effect of transition is investigated using the $\gamma-R e_{\theta}$ approach in model scale. In the applied transition model (also known as a Local Correlation-Based Transition Model LCTM) two extra field equations are inserted for turbulence modeling [14-16]. The purpose of the transport equation for turbulence intermittency $\gamma$, is to produce variable $\gamma$, which is coupled with the SST turbulence model to trigger transition in the boundary layer:

$$
\frac{\partial(\rho \gamma)}{\partial t}+\frac{\partial\left(\rho u_{j} \gamma\right)}{\partial x_{j}}=P_{\gamma}+\frac{\partial}{\partial x_{j}}\left[\left(\mu+\frac{\mu_{t}}{\sigma_{f}}\right) \frac{\partial \gamma}{\partial x_{j}}\right] .
$$

Here, $P_{\gamma}$ is the contribution from all of the source and destruction terms and $\sigma_{f}$ is a Schmidt number. The transport equation for the local transition onset momentum thickness Reynolds number $\widetilde{R e} e_{\theta t}$ captures the non-local effects of the free-stream turbulence intensity into the boundary layer:

$$
\frac{\partial\left(\rho \widetilde{R e}_{\theta t}\right)}{\partial t}+\frac{\partial\left(\rho u_{j} \widetilde{R e}_{\theta t}\right)}{\partial x_{j}}=P_{\theta t}+\frac{\partial}{\partial x_{j}}\left[\sigma_{\theta t}\left(\mu+\mu_{t}\right) \frac{\partial \widetilde{R e}_{\theta t}}{\partial x_{j}}\right] .
$$

The underlying SST $k-\omega$ model is isotropic, i.e., it predicts the Reynolds stress tensor according to the Boussinesq approximation. In other words, no individual modeling is employed for each of the normal turbulent stresses; only their sum, $k=\overline{u_{i}^{\prime} u_{i}^{\prime}} / 2$, is modeled. To account for turbulence anisotropy, the EARSM can be utilized. The idea in algebraic Reynolds stress models is to allow a nonlinear relationship between the rate of mean strain and the Reynolds stresses, but without the need of solving additional partial differential equations for each of the six Reynolds stresses. The EARSM 
relies on the two-equation formulation, but with the Reynolds stress anisotropy tensor evaluated from a linear pressure-strain model, and it forms a physically well-founded strategy among two-equation models for improved prediction of flows involving complex features, such as streamline curvature effects and system rotation $[17,18]$.

In this study, DDES (Delayed DES) is also based on the SST $k-\omega$-model [19]. DDES is a slightly modified version of the detached-eddy simulation (DES). DES and DDES reduce to a RANS model in regions where the largest turbulent fluctuations are of a smaller size to the local grid spacing. Both are hybrid RANS/LES models, and function as an LES subgrid-scale model in regions where the local turbulent phenomena are of greater size than the local grid spacing [20]. A time-accurate solution is made to resolve turbulent fluctuations. In DES, the equation for the turbulent kinetic energy $(k)$ can be written with a modified dissipation term as [21]:

$$
\rho \frac{D k}{D t}=P-\frac{\rho k^{3 / 2}}{l_{D E S}}+D
$$

where $P$ is the production of turbulence, $l_{D E S}$ is the length scale, and $D$ is the diffusion term. The DES length scale is computed as the minimum of the RANS length scale, $l_{R A N S}=\sqrt{k} / \beta^{*} \omega$, and the local resolution $\Delta$. Here, $\beta^{*}=0.09$ was a model constant. The parameter $\Delta$ is evaluated as the minimum of the local wall distance, and the grid resolution $\max \left(\Delta x_{i}\right)$, where $\Delta x_{i}$ denotes the thickness of the cell in different index directions. The DES length scale is then

$$
l_{D E S}=\min \left(C_{D E S} \Delta, l_{R A N S}\right),
$$

and the coefficient $C_{D E S}$ is computed from

$$
C_{D E S}=\left(1-F_{1}\right) C_{D E S}^{k-\varepsilon}+F_{1} C_{D E S}^{k-\omega}
$$

where the constants are $C_{D E S}^{k-\varepsilon}=0.61, C_{D E S}^{k-\omega}=0.78$, and $F_{1}$ is Menter's blending function [19]. Furthermore, when utilizing DDES, the length scale is replaced by the expression [12]

$$
l_{D D E S}=l_{R A N S}-F_{1} \max \left(0, l_{R A N S}-C_{D E S} \Delta\right) .
$$

Here, $F_{1} \rightarrow 1$ outside the boundary layer, and the length scale becomes $l_{D D E S}=C_{D E S} \Delta$ if the grid spacing permits. The DDES variant of DES aims to improve the accuracy compared to Equation (9), which has, in some instances, been observed to cause grid-induced separation.

\subsection{Mass and Energy Transfer}

A number of mass-transfer models have been suggested for cavitation [22]. Usually, the mass-transfer rate is proportional to a pressure difference from a saturated state or to a square root of that. In this study, the mass-transfer model is similar to that of Choi and Merkle [23]:

$$
\Gamma_{l}=\frac{\rho_{l} \alpha_{l} \min \left[0, p-p_{\mathrm{sat}}\right]}{\frac{1}{2} \rho_{\infty} V_{\infty}^{2}\left(L_{\mathrm{cav}} / V_{\mathrm{cav}}\right) \tau_{l}}+\frac{\rho_{g} \alpha_{g} \max \left[0, p-p_{\mathrm{sat}}\right]}{\frac{1}{2} \rho_{\infty} V_{\infty}^{2}\left(L_{\mathrm{cav}} / V_{\mathrm{cav}}\right) \tau_{g}}
$$

where $p_{\text {sat }}$ is the saturation pressure, $\rho_{\infty}$ the reference (inlet) density, and $V_{\infty}$ the corresponding velocity. The evaporation time constants were made non-dimensional using the reference length $L_{\mathrm{cav}}$ and the velocity related to cavitation $\left(V_{\text {cav }}\right)$. In some cases, such as on a propeller blade, the cavitation length and velocity differ from the reference length $L_{\text {ref }}$ and the reference velocity $\left(V_{\infty}\right)$. The time constants correspond to the parameters of the original model as $\tau_{l}=1 / C_{\text {dest }}$ and $\tau_{g}=1 / C_{\text {prod }}$. The empirical parameters of the cavitation model are calibrated in [24].

In the present method, the saturation pressure was based on the free-stream temperature, and the gas phase was assumed to be saturated (i.e., $T_{g}=T_{\text {sat }}$ ). Liquid temperature varies less because 
of the mass and energy transfer. As the gas temperature was forced to be $T_{g}=T_{\text {sat }}, q_{i g}=0$. From Equation (4), the interfacial heat transfer can be solved for the liquid phase

$$
q_{i l}=-\left(h_{g s a t}-h_{l s a t}\right) \Gamma_{g}-q_{i g}=\left(h_{g s a t}-h_{l s a t}\right) \Gamma_{l} .
$$

Using Equations (12) and (13), the interfacial transfer terms in the continuity and energy equations can be solved.

To decrease the oscillations in the solution owing to the rapid changes in the mass transfer, the mass-transfer rate was under-relaxed between the iteration cycles as $\Gamma_{l}^{n+1}=\alpha_{\Gamma} \Gamma_{l}^{*}+\left(1-\alpha_{\Gamma}\right) \Gamma_{l}^{n}$, where $\alpha_{\Gamma}=0.5$ is an under-relaxation factor, $n$ refers to the iteration cycle, and $\Gamma_{l}^{*}$ is calculated from Equation (12). For small values $\left|\Gamma_{l}^{n}\right|<0.1\left(\mathrm{~kg} / \mathrm{m}^{3} \mathrm{~s}\right)$, under-relaxation was not applied. The under-relaxation factor and the limit are quite arbitrary, although tested by numerous simulations.

\subsection{Solution Algorithm}

The solution method is a segregated pressure-based algorithm where the momentum equations are solved first, and then a pressure-velocity correction is made. The basic idea in the solution of all equations is that the mass balance is not forced at every iteration cycle, but rather the effect of the mass error is subtracted from the linearized conservation equations. A pressure correction equation was derived from the continuity equation, Equation (1), linked with the linearized momentum equation. The velocity-pressure coupling is based on the corresponding algorithm for a single-phase flow [9], and is described in more detail in $[10,25]$.

Quasi-steady simulations exploit the fact that the governing equations can yield a steady-state solution, when the equations are expressed in the coordinate system that is rotating with the propeller. Absolute velocities are used in the solution, and the rotational movement of the propeller is accounted for in the convection velocity and as source terms in the $y$ - and $z$-momentum equations as the propeller is rotating around the $x$-axis. The equations are iterated until the global force coefficients and the $L_{2}$ norms of the main variables have stabilized at a sufficiently low level. In transient simulations, the governing equations are integrated in physical time, and the whole computational domain is rotated at the propeller rate of revolution. A steady-state solution is sought within each physical time step, and the equations are iterated until the $L_{2}$ norms of the main variables have decreased by a sufficient amount, i.e., at least 2-3 orders of magnitude. Approximately one hundred inner iterations were required within each physical time step. A physical time step corresponding to 0.5 degrees of the propeller rotation was used in all transient simulations.

\section{Test Cases}

Two propellers are considered in this study: the Potsdam propeller test case (PPTC) propeller [26] and a tip loaded propeller (TLP) propeller. The former is a more conventional five-bladed propeller with moderate skew, whereas the latter is a pressure-side oriented tip blade propeller with enhanced efficiency. Photographs of the propellers are shown in Figure 2. The principal data of the studied propellers is given in Table 1 for the PPTC and in Table 2 for the TLP.

The advance coefficient and cavitation number are defined as

$$
J=\frac{V_{A}}{n D} \text { and } \sigma_{n}=\frac{p-p_{\text {sat }}}{\frac{1}{2} \rho(n D)^{2}},
$$

respectively, where $V_{A}$ is the propeller speed of advance, $n$ the propeller rate of revolutions, $D$ the propeller diameter, $p$ is the pressure, $p_{\text {sat }}$ the saturation pressure, and $\rho$ the fluid density. The thrust and torque of the propeller are nondimensionalized as

$$
K_{T}=\frac{T}{\rho n^{2} D^{4}} \text { and } K_{Q}=\frac{Q}{\rho n^{2} D^{5}}
$$


respectively, where $T$ denotes the thrust and $Q$ the torque of the propeller. The open-water efficiency of the propeller is $\eta_{0}=J /(2 \pi) K_{T} / K_{Q}$. The Reynolds number is defined as

$$
\operatorname{Re}=\frac{\rho c_{0.7} \sqrt{V_{A}^{2}+(0.7 \omega D / 2)^{2}}}{\mu},
$$

where $\omega=2 \pi n$, and $c_{0.7}$ denotes the chord length at $r / R=0.7$ radius.

Table 1. Main geometric parameters of the Potsdam propeller test case (PPTC) propeller [26].

\begin{tabular}{lc}
\hline Diameter $[\mathrm{m}]$ & 0.250 \\
Pitch ratio at $r / R=0.7$ & 1.635 \\
Chord at $r / R=0.7$ & 0.417 \\
EAR & 0.779 \\
Skew $\left[^{\circ}\right]$ & 18.837 \\
Hub ratio & 0.300 \\
Number of blades & 5 \\
Rotation & Right-handed \\
\hline
\end{tabular}

Table 2. Main geometric parameters of the tip loaded propeller (TLP) propeller.

\begin{tabular}{lc}
\hline Diameter $[\mathrm{m}]$ & 0.250 \\
Pitch ratio at $r / R=0.7$ & 1.465 \\
EAR & 0.594 \\
Number of blades & 5 \\
Rotation & Right-handed \\
\hline
\end{tabular}

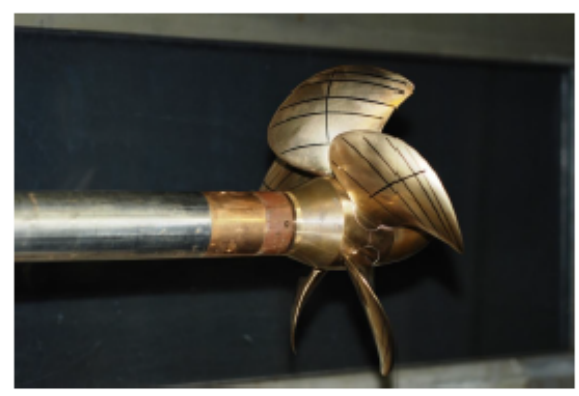

(a) PPTC.

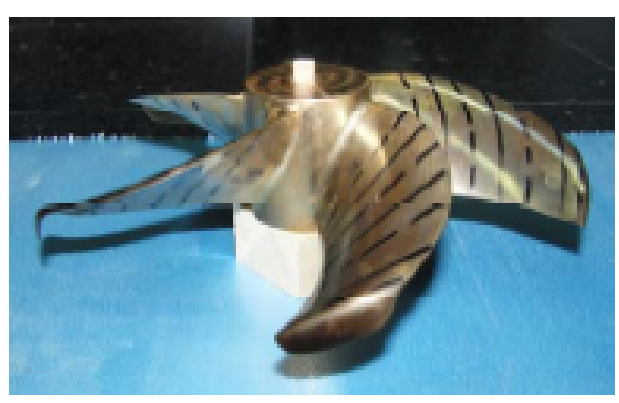

(b) TLP.

Figure 2. Photographs of the investigated propellers. The black circles on the blades show the reference radii. Photograph of PPTC taken from Ref. [26] and reproduced with permission from Lars Lübke (SVA). Photograph of TLP reproduced with permission from Michael Brown (ONR).

The investigated conditions for the cavitating cases are listed in Table 3 for each propeller. We study the cavitating conditions for the PPTC at five different Reynolds numbers, and for the TLP at two different Reynolds numbers. Two different cases are studied with the PPTC using different $J$ and $\sigma_{n}$, given in Table 3 . The Case 1 yields mostly steady vortex and sheet cavitation patterns, whereas the Case 2 exhibits unsteady cavitation phenomena on the propeller blades and in the slipstream. The model-scale conditions were chosen to match the experimental conditions (for PPTC Case 1, this corresponds to $R e_{2}$; the $R e_{1}$ was chosen to assess also a lower Reynolds number in model scale for this operating point), and the full-scale conditions were selected to ensure a sufficiently high Reynolds number. The reference length and velocity scales related to the Merkle cavitation model, discussed in Section 2.3, were here the chord and the speed at $r / R=0.7$ radius. We used the same values for the empirical parameters in the cavitation model in model- and full-scale simulations, and they were $C_{\text {dest }}=C_{\text {prod }}=369$. 
Table 3. Test conditions for the cavitating cases.

\begin{tabular}{lccc}
\hline Quantity & PPTC, Case 1 & PPTC, Case 2 & TLP \\
\hline$J$ & 1.019 & 1.269 & 0.729 \\
$\sigma_{n}$ & 2.024 & 1424 & 4.092 \\
$K_{T}$ & 0.374 & 0.206 & 0.493 \\
$n$ [rps] & 25.0 & 25.0 & 15.0 \\
$\operatorname{Re}$, model scale & $R e_{1}=5.0 \times 10^{5}$, & $1.4 \times 10^{6}$ & $9.7 \times 10^{5}$ \\
& $R e_{2}=1.3 \times 10^{6}$ & $3.0 \times 10^{7}$ & $5.7 \times 10^{7}$ \\
\hline Re, full scale & $2.9 \times 10^{7}$ & 3 & \\
\hline
\end{tabular}

The Case 1 for the PPTC is studied in model scale using the SST, the transition model (TM) and the EARSM. This condition is studied in full scale using SST. The Case 2 for the PPTC is studied employing URANS (unsteady RANS) with transient SST simulations and DDES in model scale and full scale. The TLP is studied in model scale using the SST and the TM. This condition is studied in full scale using SST.

\subsection{Computational Setup}

Two grids were constructed for both propellers: a model-scale grid and a full-scale grid. The model-scale grids were generated to simulate the experimental set-ups. The computational grids for the full scale simulations were scaled from the model scale grids, and additional cells were clustered close to solid surfaces to ensure that $y^{+} \approx 1$ for the simulations. The scaling factor for PPTC was 12 , and for the TLP this was 26.

The computational domain is shown in Figure 3 for the PPTC propeller. Corresponding design for the computational domain was used for TLP propeller. The calculations were performed with three grid densities. On the coarse grid level, every second point in all directions is removed compared to the finer level grid. A solution on the coarse grid is used as an initial guess for the computations performed on the next finer grid level. The fine computational grid used for the PPTC propeller consists of $\sim 6.5$ million cells in 36 grid blocks, the medium grid consists of 0.8 million cells, and the coarse grid of 0.1 million cells. The fine grid for the TLP consists of roughly 10 million cells in 45 grid blocks, the medium grid consists of 1.3 million cells, and the coarse grid of 0.2 million cells. The different grids on the suction sides of the propeller blades are shown in Figure 4. The surface grid on the pressure sides of the blades is similar. A similar grid for the PPTC was used before [10,25], where the numerical simulations with the hybrid RANS/LES model showed very good agreement also with respect to the local flow LDV (laser Doppler velocimetry) measurements in the propeller wake. The grid density in the wake region was further increased for this study. Due to the symmetric nature of the problem of a propeller operating in uniform inflow, only one blade is modeled. The blades, hub, and shaft are modeled as no-slip rotational surfaces. A velocity boundary condition is applied at the inlet and a pressure boundary condition is applied at the outlet. A slip boundary condition is applied at the simplified cavitation tunnel walls.

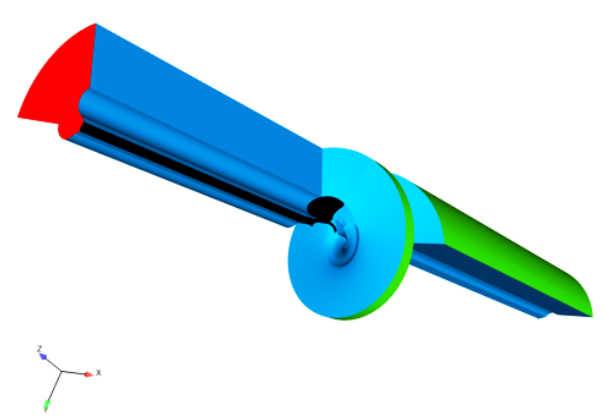

Figure 3. Perspective view of the computational domain for the PPTC. The propeller, hub, and shaft are colored with black. The inlet face is colored with red, the cyclic boundaries with blue, and the simplified tunnel wall with green. 


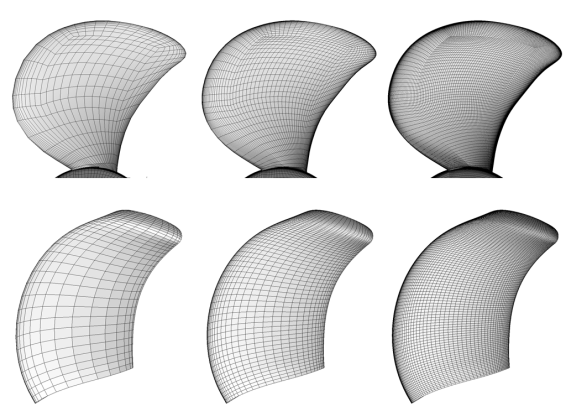

Figure 4. Grid density on the suction sides of the PPTC (top row) and TLP (bottom row) propeller blades. Coarse (left), medium (middle), and fine (right) model-scale grids shown.

The whole computational domains are considered as rotating with the given rate of rotation. The inflow velocity is set based on the advance numbers of the propeller, and the background pressure level is set based on the cavitation number. Low turbulent intensity (1\%) and eddy viscosity $\left(\mu_{t} / \mu=10\right)$ ratio were used as initial conditions in all calculations. We note here that the sensitivity of the transition model to the initial turbulence field quantities in the model-scale cavitating propeller performance predictions needs be assessed in the future. The inlet is located roughly five propeller diameters upstream of the propeller, and the outlet is located ten diameters downstream of the propeller. For the PPTC propeller, the radius of the whole computational domain is $0.3385 \mathrm{~m}$ in model scale, which corresponds to the cross-sectional area of the experimental cavitation tunnel. The radius of $0.7 \mathrm{~m}$ in model scale was used for the computational domain for the TLP, which was larger in cross-sectional area than in the experimental tank. Typically, a steady-state case requires around one day of computational time on fine grid resolution with approximately 20 CPUs, whereas an unsteady case requires approximately 3 days with the similar machine set-up to reach one full revolution.

\section{Validation of the Numerical Method}

In this section, we perform a validation study of our numerical simulations in terms of the predicted global forces and cavitation patterns. Also, a grid and time step sensitivity study is carried out. We consider both the model- and full-scale conditions in the validation. Section 4.1 concerns the global performance characteristics of the propellers, Section 4.2 the cavitation observation, and Section 4.3 shows a grid and time step sensitivity analysis.

\subsection{Global Forces}

The open-water characteristics in non-cavitating conditions for the propellers are shown in Figure 5. We produced the open-water curves for the PPTC from $J=0.5 \ldots 1.6$ and for the TLP from $J=0.5 \ldots 1.2$ with the SST $k-\omega$ turbulence model in model-scale (MS) conditions. Selected points are also simulated with the EARSM and $\gamma-R e_{\theta}$ models, as well as in full-scale (FS) conditions. The SST and EARSM simulations in model scale capture the propeller open-water performance with good accuracy for both propellers. EARSM predicts slightly lower torque for the TLP propeller than the SST model. The transition simulations predict 2-3\% higher thrust coefficient than the SST in model scale for the propellers, at the investigated points. The transition simulations, as well the full-scale simulations, yield a slightly lower torque coefficient, and consequently, a little higher efficiency for the propeller. The predicted thrust coefficients and their comparison with the experimental values are given in Table 4. Greater differences with respect to the experiments are seen in the unsteady case, where it is also noteworthy that the model- and full-scale simulations both gave the same thrust coefficient. 


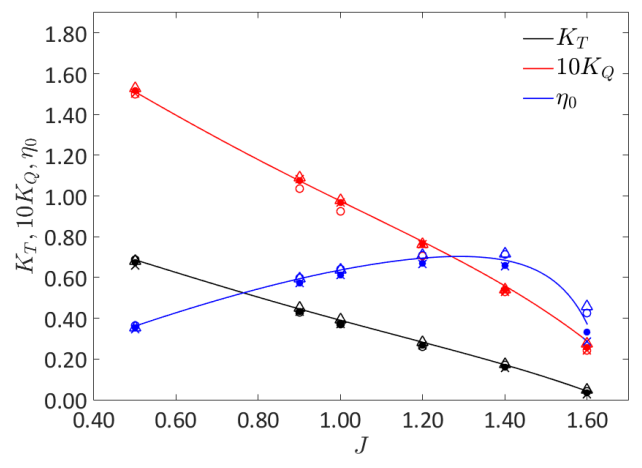

(a) PPTC.

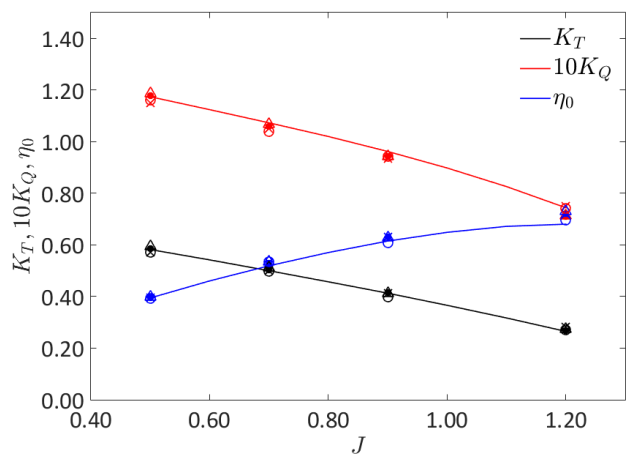

(b) TLP.

Figure 5. Open-water characteristics for the propellers. Solid lines: experimental results; $\bullet$ symbols denote corresponding $k-\omega$ model-scale simulations; $\times$ symbols denote EARSM model-scale simulations; $\triangle$ symbols denote $\gamma-R e_{\theta}$ model-scale simulations; ○ symbols denote $k-\omega$ full-scale simulations.

The thrust coefficients in the cavitating conditions are compared to the model-scale experiments in Table 4. All steady-state RANS simulations yield results within $4 \%$ of the experimental values. A greater difference is seen at the PPTC Case 1 conditions for the lowest investigated Re, where both the SST and TM underpredict the propeller thrust. The SST and TM give similar results for the TLP. The results at full-scale Reynolds numbers tend to have the least deviation with respect to the experiments. For the transient simulations (PPTC Case 2), the model-scale DDES gives a slightly lower and the full-scale DDES a slightly higher thrust coefficient. The URANS simulations yield a greater difference when compared to the experiments. The SST predicts a smaller thrust value than the DDES in model scale, and a higher in full scale. The TM significantly underpredicts the propeller thrust. Although not shown here, we note that the TM gave a very steady time history of the propeller global forces, with next to no fluctuations in the $K_{T}$ and $K_{Q}$ values. The simulations with the other turbulence modeling approaches (both URANS SST and the DDES in model- and full-scale), then again, predicted a more unsteady cavitation on the blades and consequently also highly oscillating global force time histories. See also Sections 4.2 and 4.3 .

Table 4. Predicted thrust coefficients in cavitating conditions.

\begin{tabular}{lcc}
\hline Case & $\begin{array}{c}\text { Thrust } \\
\text { Coefficient }\end{array}$ & $\begin{array}{c}\text { Difference to } \\
\text { Experiments }\end{array}$ \\
\hline PPTC, Case $1, R e_{1}=5.0 \times 10^{5}, \mathrm{SST}$ & 0.360 & $-4 \%$ \\
PPTC, Case $1, R e_{1}=5.0 \times 10^{5}, \mathrm{TM}$ & 0.361 & $-4 \%$ \\
PPTC, Case $1, R e_{2}=1.3 \times 10^{6}, \mathrm{SST}$ & 0.369 & $-1 \%$ \\
PPTC, Case $1, R e_{2}=1.3 \times 10^{6}, \mathrm{TM}$ & 0.364 & $-3 \%$ \\
PPTC, Case $1, R e=2.9 \times 10^{7}, \mathrm{SST}$ & 0.373 & $0 \%$ \\
\hline PPTC, Case $2, R e=1.4 \times 10^{6}, \mathrm{SST}$ & 0.195 & $-5 \%$ \\
PPTC, Case $2, R e=3.0 \times 10^{6}, \mathrm{TM}$ & 0.183 & $-12 \%$ \\
PPTC, Case $2, R e=1.4 \times 10^{6}, \mathrm{DDES}$ & 0.204 & $-1 \%$ \\
PPTC, Case $2, R e=3.0 \times 10^{7}, \mathrm{SST}$ & 0.212 & $3 \%$ \\
PPTC, Case $2, R e=3.0 \times 10^{7}, \mathrm{DDES}$ & 0.208 & $1 \%$ \\
\hline TLP, $R e=9.7 \times 10^{5}, \mathrm{SST}$ & 0.503 & $2 \%$ \\
TLP, $R e=9.7 \times 10^{5}, \mathrm{TM}$ & 0.505 & $2 \%$ \\
TLP, $R e=5.7 \times 10^{7}, \mathrm{SST}$ & 0.500 & $1 \%$ \\
\hline
\end{tabular}

\subsection{Cavitation Observation}

The model-scale cavitation patterns of the propellers are compared to the experimental observations. We compare both the near-blade cavitation and the overall cavitation patterns also 
in the wake. The cavitation extents based on the experiments are compared to the simulations with the different turbulence modeling approaches. The cavitation is visualized in the numerical results by the iso-surface of the void fraction value 0.1. In this section, all model-scale results for PPTC Case 1 conditions are those with the higher Reynolds number, that is, $R e_{2}=1.3 \times 10^{6}$.

The model-scale cavitation patterns of the PPTC Case 1 are compared in Figures 6 and 7. The former figure shows the cavitation type near the blades, and the latter figure shows the cavitation structures also in the propeller slipstream. The experiments are compared to the results obtained with the different turbulence models. The propeller has strong tip vortex and hub vortex cavitation, which is visible in the experiments and in the simulations. The mean shape and extent of the root cavitation, as well as the tip and hub vortex cavitation, are captured well. The tip and hub vortex cavitation extending far behind the propeller are captured exceptionally well, as shown in Figure 7. Also, the modal shapes of the cavitating tip vortex are qualitatively well captured. Streak cavitation was observed in the experiments at several radial locations near the leading edge of the blade, whereas the simulation predicts sheet cavitation at the leading edge. The global cavitation is predicted similarly with each of the utilized turbulence models, although the transition model yields a bit narrower hub cavitating hub vortex. Little difference is seen between the utilized turbulence modeling approaches for the PPTC Case 1 conditions.

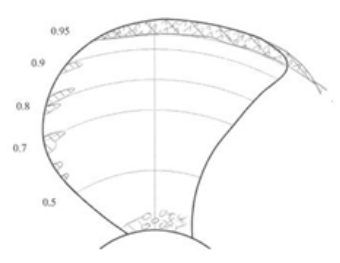

(a) EFD.

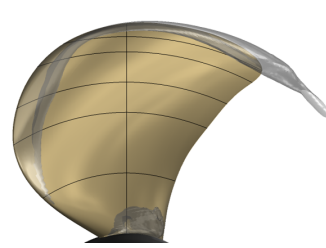

(b) CFD: SST.

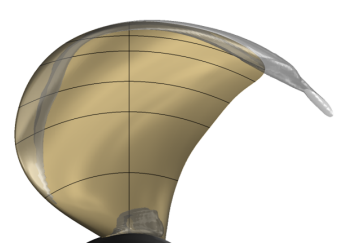

(c) CFD: EARSM.

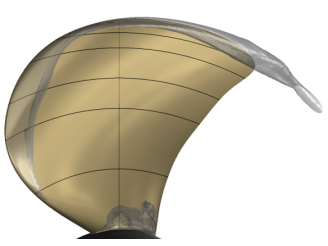

(d) CFD: TM.

Figure 6. Comparison of model-scale near-blade cavitation observation for the PPTC Case 1. The EFD result is given in [27], and reproduced with permission from Lars Lübke (SVA).

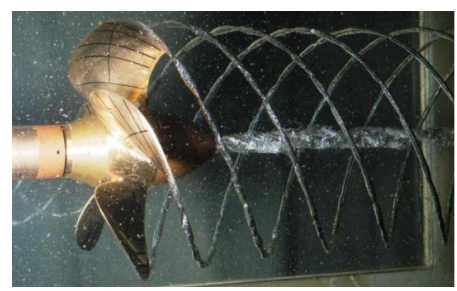

(a) EFD.

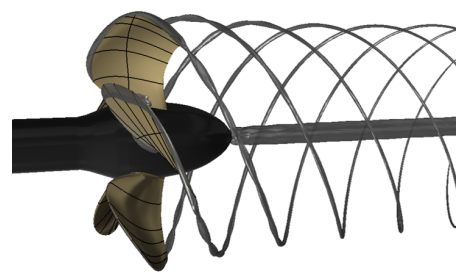

(c) CFD: EARSM.

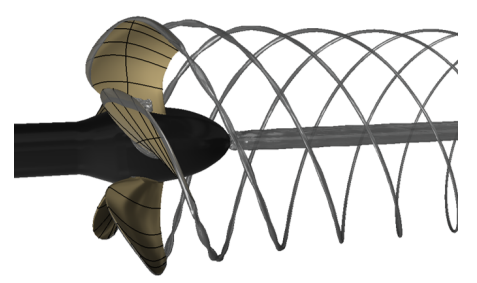

(b) CFD: SST.

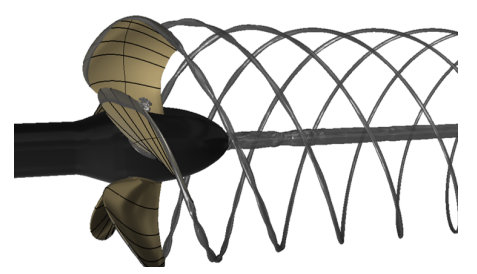

(d) CFD: TM.

Figure 7. Comparison of model-scale cavitation patterns for the PPTC Case 1. The photograph from experiments is given in [26], and reproduced with permission from Lars Lübke (SVA).

The model-scale cavitation patterns of the PPTC Case 2 are compared in Figures 8 and 9, the former figure showing the cavitation type near the blades, and the latter the cavitation structures in the propeller slipstream. Also here, the experiments are compared to the results obtained with the different turbulence models. The propeller has a finer tip vortex cavitation and stronger root cavitation. The root cavitation extends past the blade trailing edge and sheds unsteady vapor structures in the 
wake. Bubble cavitation on the blade is observed in the experiments, covering roughly the entire blade between the root cavitation zone and the tip. The SST simulations and the DDES predict larger root cavitation extent than is visible in the experiments; additionally, the bubble cavitation on the blade appears as a fine sheet cavity. The TM shows a quite extensive root cavitation and a small cavity spot on the blade. The tip vortex cavitation incepts at roughly the same location in the experiments and in the simulations in Case 2. Unsteady shedding of the root cavitation occurs in the experiments in Case 2 , subsequently transforming into cloudy cavitation in the propeller slipstream. The SST simulations and the DDES also predict unsteady shedding of cavitation structures from the root, and these attain a slightly more visible and organized form (Figure 9). TM simulations for PPTC Case 2 fail to predict the slipstream cavitation; the transition model also predicted a notably lower $K_{T}$ for this condition (cf. Table 4). The propeller also experiences pressure side cavitation at this operating condition, which is further assessed in Section 5.

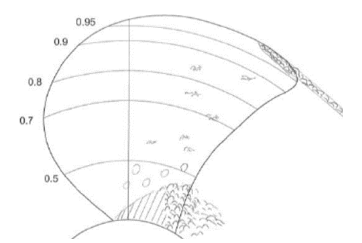

(a) EFD.

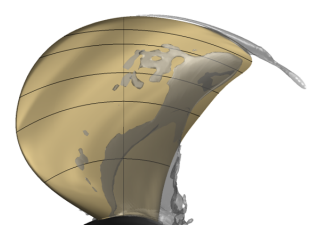

(b) CFD: SST.

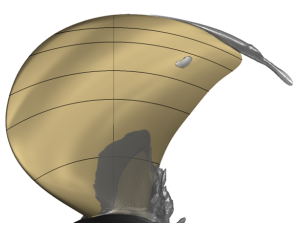

(c) CFD: TM.

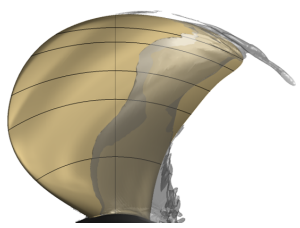

(d) CFD: DDES.

Figure 8. Comparison of model-scale near-blade cavitation observation for the PPTC Case 2. The EFD result is given in [26], and reproduced with permission from Lars Lübke (SVA).

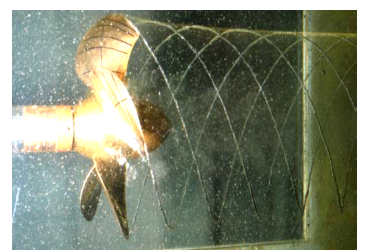

(a) EFD.

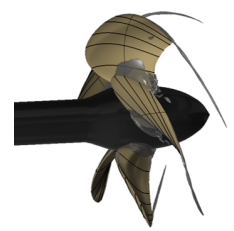

(c) CFD: TM.

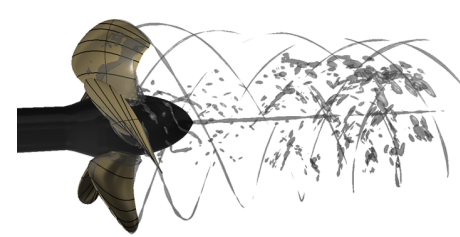

(b) CFD: SST.

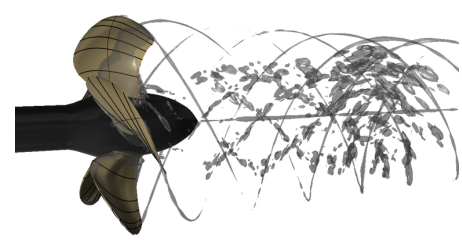

(d) CFD: DDES.

Figure 9. Comparison of model-scale cavitation patterns for the PPTC Case 2. The photograph from experiments is given in [26], and reproduced with permission from Lars Lübke (SVA).

The model-scale cavitation extent of the TLP propeller is compared in Figure 10 for cavitation patterns near the blades, and in Figure 11 for overall cavitation extent of the propeller. We show the experimental results together with those obtained with the different turbulence models. The propeller has sheet cavitation upward of $0.9 \mathrm{R}$ toward the tip which incepts at the leading edge. The simulations predict this, although the sheet starts at a slightly lower radius than in the experiments. The sheet cavitation covers almost the entire chord of the blade, and turns cloudy close to the trailing edge. The root cavitation is observed neither in the experiments nor the simulations. The upper part of the sheet cavity transforms into a cavitating tip vortex as it departs the blade. The cavitating tip vortex is relatively fine and visible both in the experiments as well as in the simulations. As we see in Figure 11, the cavitating tip vortex extends far in the propeller wake, and this behavior is also captured well with the numerical simulations with all utilized turbulence models. Also, the secondary fine tip vortex cavitation due to the end plate is captured in the CFD simulations, although it quickly diminishes 
after departing the blade. Again, the global cavitation is predicted similarly with each of the utilized turbulence models.

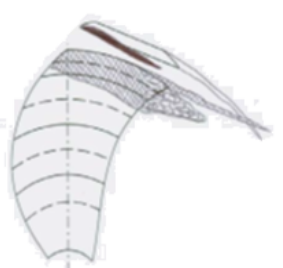

(a) EFD.

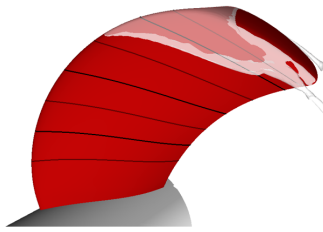

(b) CFD: SST.

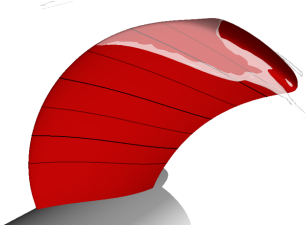

(c) CFD: EARSM

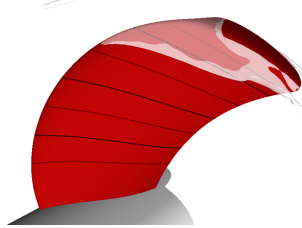

(d) CFD: TM.

Figure 10. Comparison of near-blade cavitation patterns for the TLP.

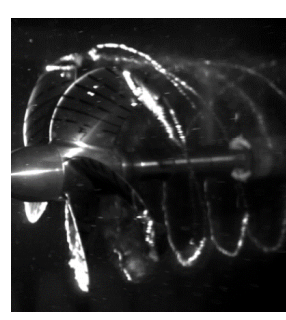

(a) EFD.

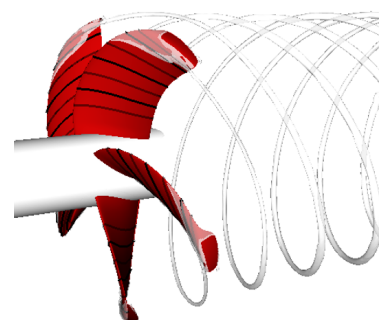

(b) CFD: SST.

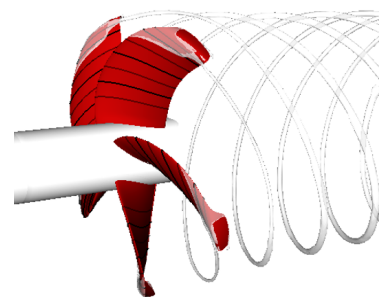

(c) CFD: EARSM.

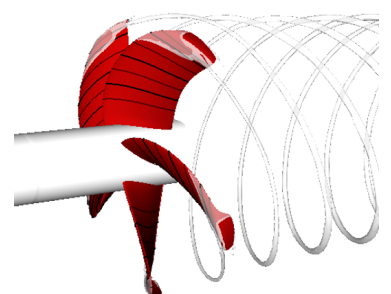

(d) CFD: TM.

Figure 11. Comparison of model-scale cavitation patterns for the TLP.

\subsection{A Grid and Time Step Sensitivity Study in Model-and Full-Scale Conditions}

Next, we carry out a grid sensitivity investigation for the PPTC Case 1 . We consider the modeland full-scale simulations at three different numerical grid densities. The propeller performance characteristics in model- and full-scale are given in Table 5 . The simulated performance characteristics are compared to the model test results.

Table 5. Predicted thrust and torque coefficients with the different grid densities for PPTC Case 1. The percentage difference to the model-scale values from the experiments is given in parentheses.

\begin{tabular}{lccc}
\hline Case & $\begin{array}{c}\text { Thrust } \\
\text { Coefficient, } \boldsymbol{K}_{\boldsymbol{T}}\end{array}$ & $\begin{array}{c}\text { Torque } \\
\text { Coefficient, } 10 \boldsymbol{K}_{\boldsymbol{Q}}\end{array}$ & $\begin{array}{c}\text { Open-water } \\
\text { Efficiency, } \boldsymbol{\eta}_{\mathbf{0}}\end{array}$ \\
\hline Experiments (model scale) & 0.374 & 0.970 & 0.625 \\
\hline Coarse grid, model scale & $0.343(-9 \%)$ & $0.874(-11 \%)$ & $0.637(3 \%)$ \\
Medium grid, model scale & $0.352(-6 \%)$ & $0.907(-7 \%)$ & $0.630(1 \%)$ \\
Fine grid, model scale & $0.369(-1 \%)$ & $0.950(-2 \%)$ & $0.629(1 \%)$ \\
\hline Coarse grid, full scale & $0.338(-11 \%)$ & $0.833(-16 \%)$ & $0.658(5 \%)$ \\
Medium grid, full scale & $0.358(-4 \%)$ & $0.889(-9 \%)$ & $0.654(4 \%)$ \\
Fine grid, full scale & $0.373(-0 \%)$ & $0.940(-3 \%)$ & $0.644(3 \%)$ \\
\hline
\end{tabular}

We note that the finest grids produce results that are within few per cents of the experimental values. The full-scale simulations yield a bit higher thrust coefficient, a slightly lower torque coefficient, and consequently a higher open-water efficiency. The propeller efficiency, again, does not show as great dependency on the utilized grid density in either model- or full-scale as the global forces do. Both the model- and full-scale performance characteristics converge monotonically toward the experimental values.

Figure 12 displays the near-blade cavitation results, and Figure 13 shows the overall cavitation extent obtained with different numerical grid densities. Furthermore, we compare the pressure in the wake in Figure 14 with the three grids. We evaluated the pressure coefficient, $C_{p}=$ $2\left(p-p_{\infty}\right) /\left(\rho_{\infty} n^{2} D^{2}\right)$, in the wake at the track of the tip vortex. 


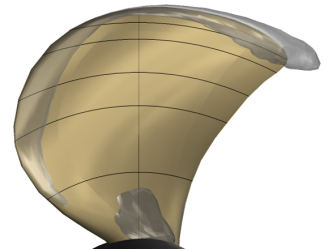

(a) Model-scale: Coarse grid.

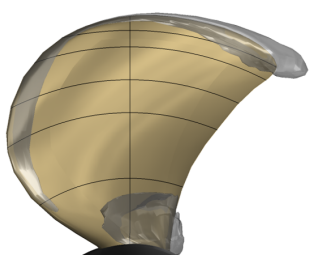

(d) Full-scale: Coarse grid.

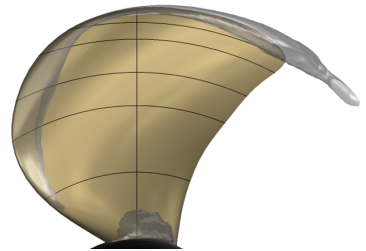

(b) Model-scale: Medium grid.

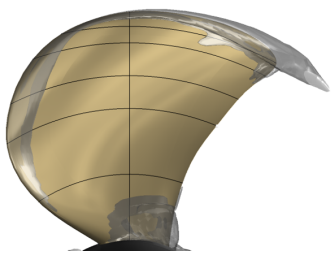

(e) Full-scale: Medium grid.

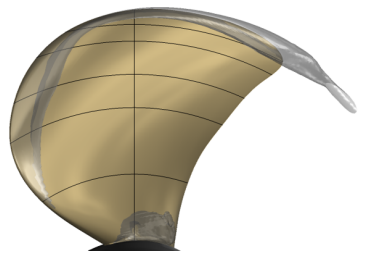

(c) Model-scale: Fine grid.

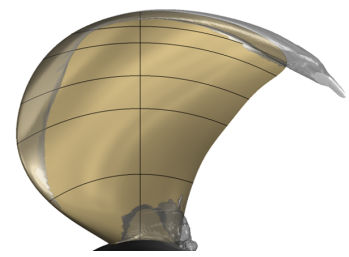

(f) Full-scale: Fine grid.

Figure 12. Comparison of near-blade cavitation results with different grid densities. PPTC Case 1 conditions, results with the SST $k-\omega$ model.

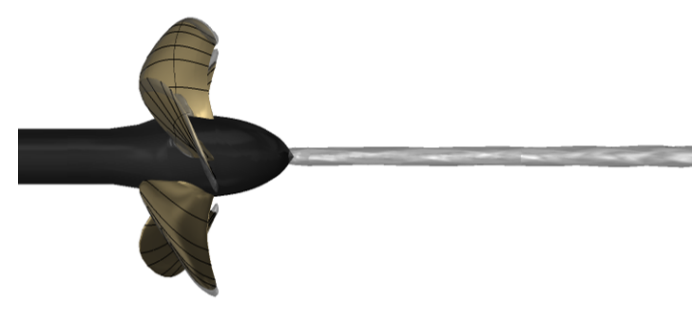

(a) Coarse grid.

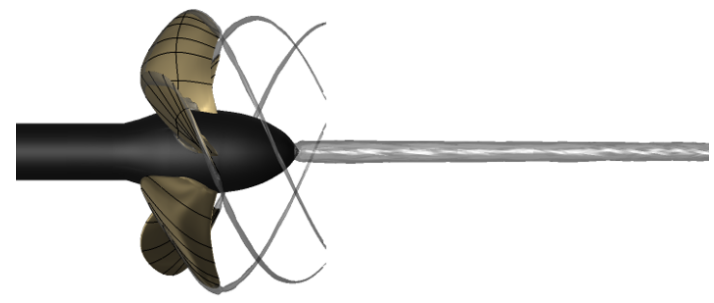

(b) Medium grid.

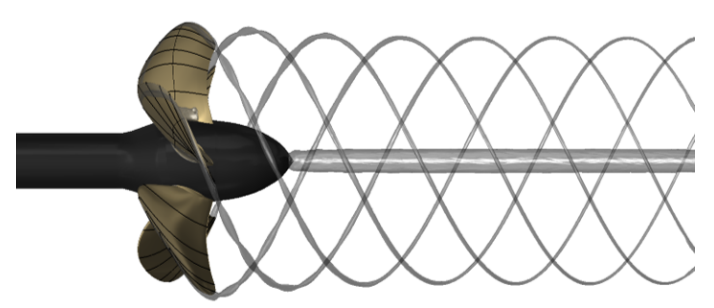

(c) Fine grid.

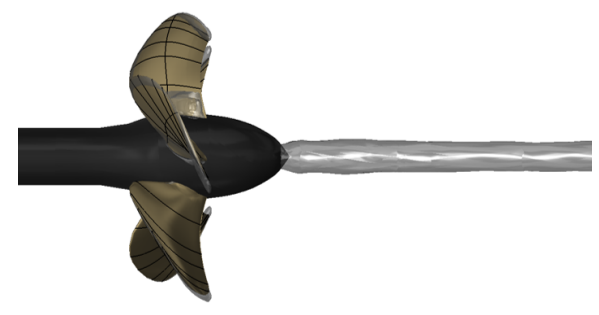

(d) Coarse grid.

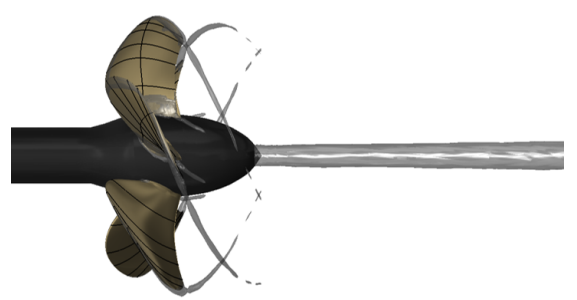

(e) Medium grid.

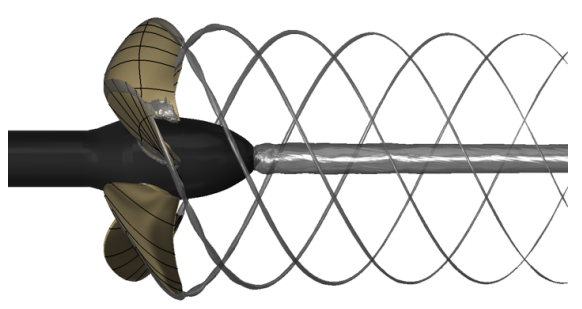

(f) Fine grid.

Figure 13. Comparison of cavitation patterns for the PPTC propeller with different grid densities. On the left: model-scale simulations. On the right: full-scale simulations. 

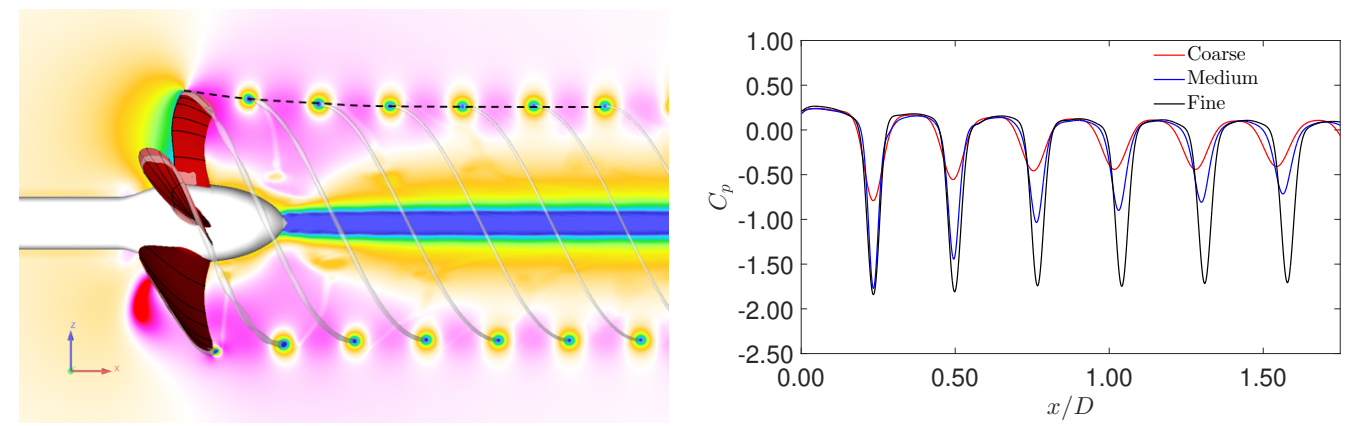

Figure 14. Pressure coefficient in the track of the tip vortex with different grid densities for the PPTC Case 1 conditions. Model-scale SST simulations. The figure on the left shows the fine grid SST simulations and the track as dashed line along which the $C_{p}$ is evaluated.

The cavitation for PPTC Case 1 near the propeller blades is similar to the different utilized grids. All grids predict sheet cavitation at the leading edge which then transforms to the tip vortex cavitation. The root cavitation generally grows in extent as the grid is refined, but otherwise the near-blade cavitation is mostly independent of the utilized grid densities. We observe greater differences in the results between the different grids in the propeller wake. The coarse grid simulations yield a very limited vortex cavitation, as the tip vortex cavitation seizes just after the blade trailing edge. We do not, however, observe such a clear influence of the grid density on the hub vortex cavitation. The medium grid simulations give the hub vortex cavitation that match better the fine grid results, and the experiments, but the tip vortex cavitation extends less than one diameter in the axial direction. The modal shapes are also barely distinguishable in the medium grid solution. Figure 14 shows the decay in the pressure minima in model scale is notably greater for the coarse and medium grids as the axial distance from the propeller increases. The pressure coefficient in the first vortex core for the coarse grid is significantly less than the vapor pressure, and therefore the tip vortex cavitation terminates just after the blades. For the medium grid, the first tip vortex core on the curve maintains a low $C_{p}$ level, and we observed a cavitating vortex in Figure 13b. Beyond this, the pressure coefficient decreases and the tip vortex cavitation disappears.

The skin-friction and pressure coefficients for the different grid densities are compared in Figures 15 and 16 for model- and full-scale conditions for the PPTC, respectively. We defined the skin-friction coefficient as $C_{f}=2\left|\tau_{i j}\right| /\left(\rho_{\infty} n^{2} D^{2}\right)$. In these figures, the coefficients are plotted on both sides of the blades, extracted from the radius $r / R=0.7$. Furthermore, the skin-friction and pressure coefficients are visualized on the blades in Figures 17 and 18 for the model- and full-scale conditions, together with surface-flow LIC (line integral convolution). The skin-friction coefficient shows greater sensitivity to the utilized grid density, especially on the suction sides of the blades. This is expected, as the wall $y^{+}$values deteriorate as the grid resolution decreases. The pressure coefficient, however, exhibits much less variation between the different grid densities both in modeland full-scale simulations. The pressure coefficients at the $r / R=0.7$ practically overlap each other in Figures 15 and 16 for the most part of the blade. The increase in the pressure after the leading edge (LE) sheet cavity closure is not visible in the coarse grid simulations, whereas the medium and fine grid simulations show clearly elevated $C_{p}$. The coarse grid $C_{p}$ is very smooth in both modeland full-scale conditions, as we see also in Figures 15 and 16. The fine grid $C_{p}$ shows quite sharp a jump at the cavity closure line in model- and full-scale conditions. Significantly lower skin-friction values are seen underneath the sheet cavitation near the LE. The $C_{f}$ shows also quite sharp an increase after the cavity closure in the fine grid simulations, being smoother for the coarser grid solutions. In addition, the surface flow underneath the cavity deviates drastically from the typical (turbulent) circular paths in the model-scale medium and fine grid solutions. In model-scale conditions, the surface flows show steeper turns back toward the LE than in full scale. The coarse grid solutions fails to show similar phenomena. Interestingly, judging by the surface flow visualization, the medium grid solution in model scale predicts the cavity closure line a bit farther from the LE than the fine grid 
solution. The opposite is noted in full-scale, where the fine grid solution now predicts the closure line farther from the LE than the medium grid solution (we note that this behavior was not so clear in the blade $C_{p}$ or $C_{f}$ distributions, Figures 15 and 16). In full scale, the surface flow underneath the sheet cavity is oriented differently between the medium and fine grid solutions, as it turns upward toward the tip closer to the LE with the medium grid.
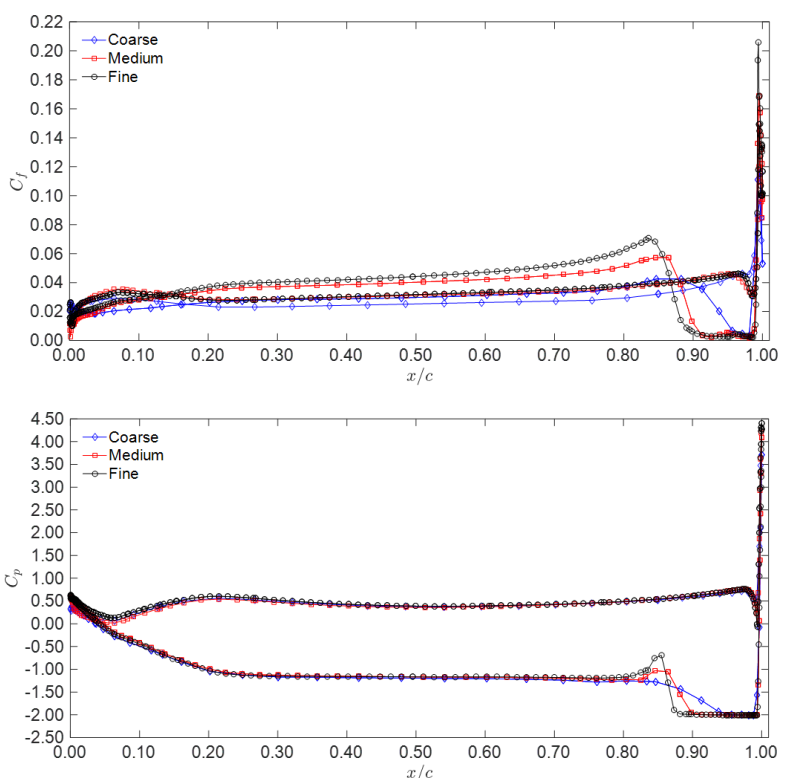
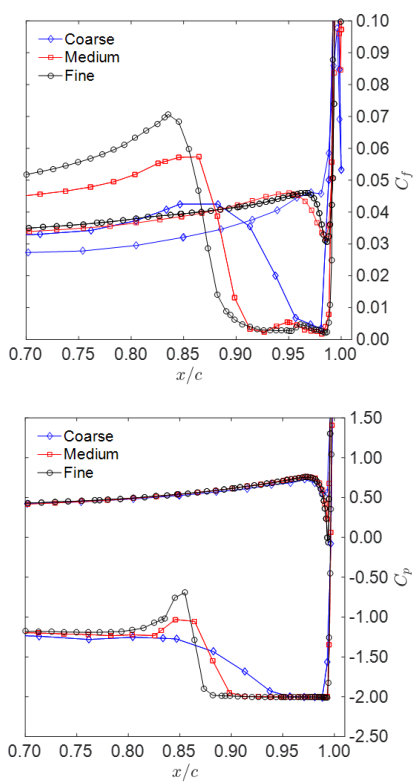

Figure 15. Comparison of skin-friction and pressure coefficient distributions at the radius $r / R=0.7$ on the blades. PPTC Case 1 conditions in model scale. The results are from the three grid densities. The TE is at $x / c=0$ and the LE at $x / c=1$.
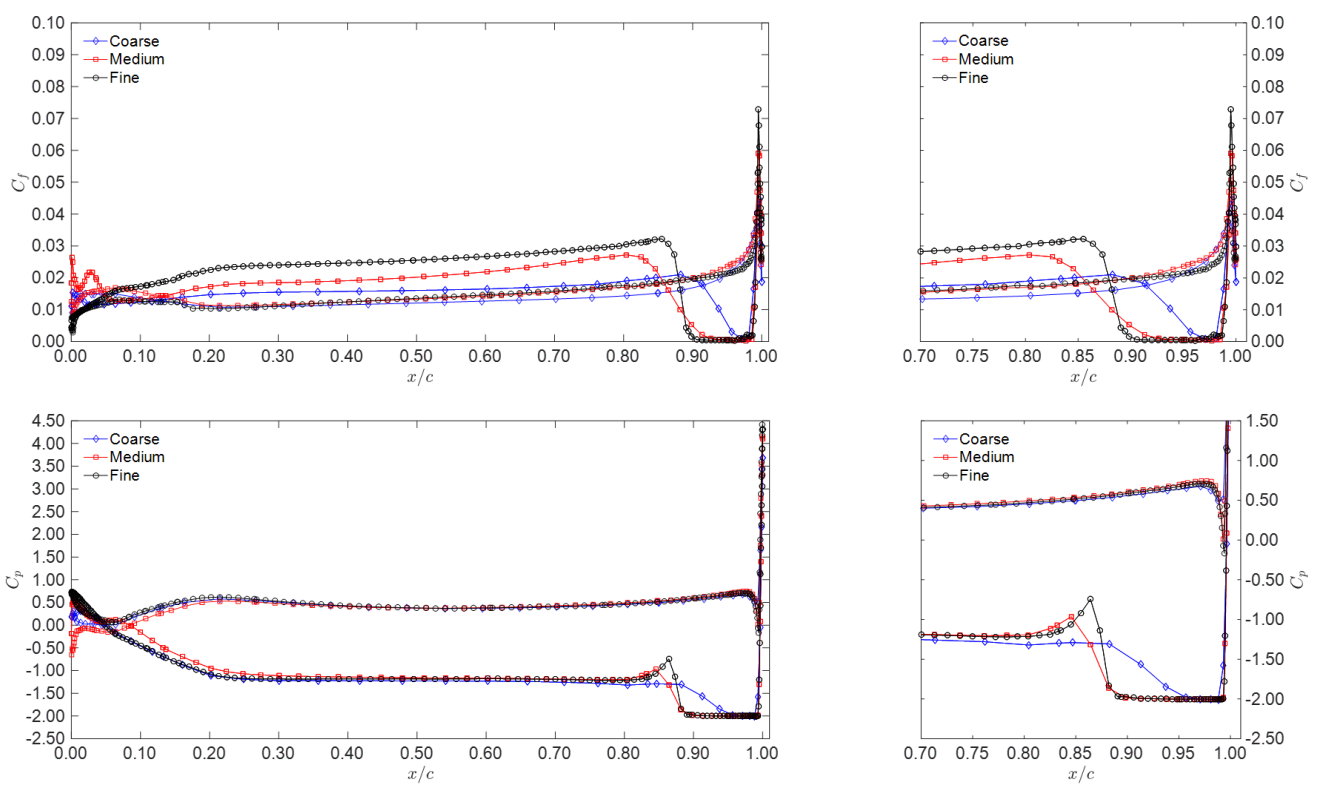

Figure 16. Comparison of skin-friction and pressure coefficient distributions at the radius $r / R=0.7$ on the blades. PPTC Case 1 conditions in full scale. The results are from the three grid densities. The TE is at $x / c=0$ and the $\mathrm{LE}$ at $x / c=1$. 


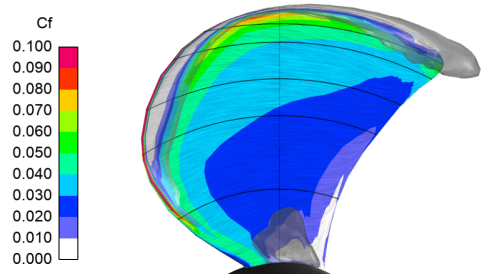

(a) Coarse grid, $C_{f}$.
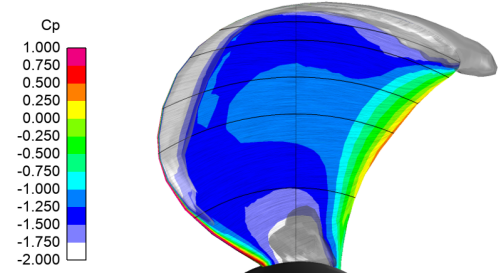

(d) Coarse grid, $C_{p}$.

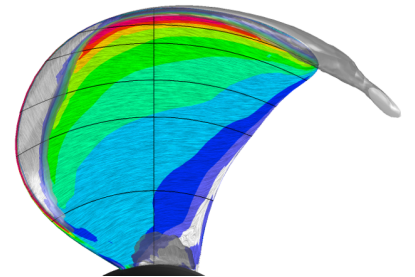

(b) Medium grid, $C_{f}$.

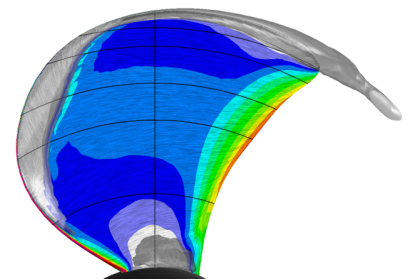

(e) Medium grid, $C_{p}$.

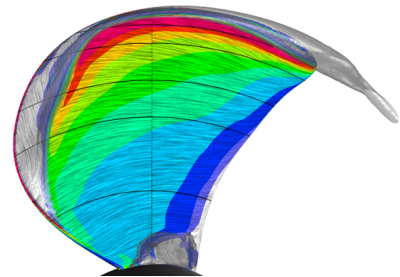

(c) Fine grid, $C_{f}$.

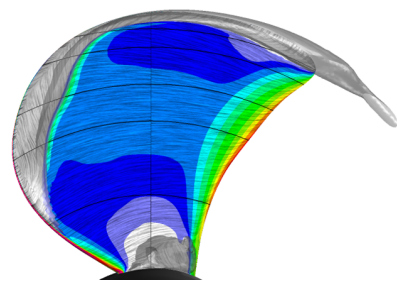

(f) Fine grid, $C_{p}$.

Figure 17. Comparison of near-blade cavitation results with different grid densities. Top row: skin-friction coefficient. Bottom row: pressure coefficient. Model-scale simulations for PPTC Case 1.

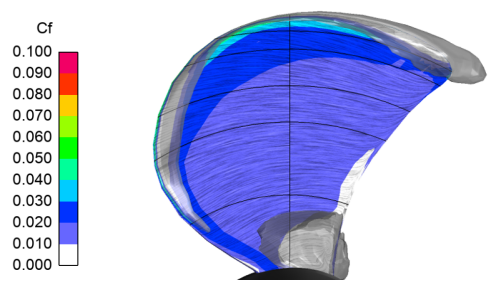

(a) Coarse grid, $C_{f}$.
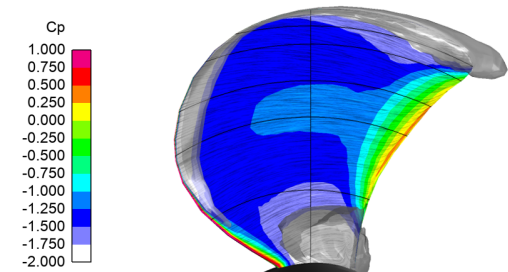

(d) Coarse grid, $C_{p}$.

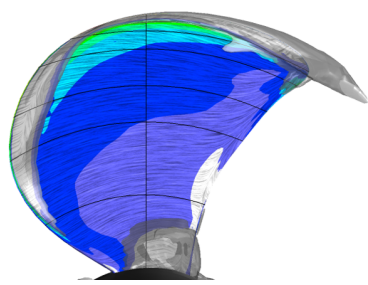

(b) Medium grid, $C_{f}$.

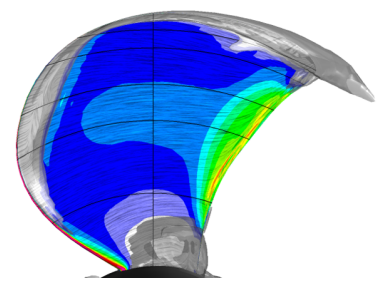

(e) Medium grid, $C_{p}$.

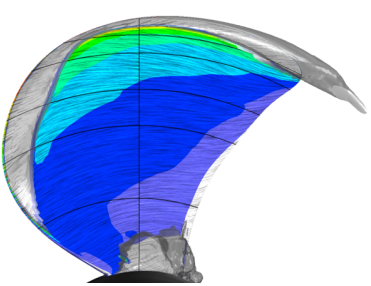

(c) Fine grid, $C_{f}$.

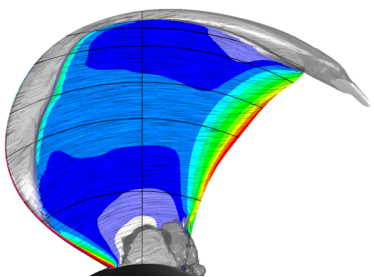

(f) Fine grid, $C_{p}$.

Figure 18. Comparison of near-blade cavitation results with different grid densities. Top row: skin-friction coefficient. Bottom row: pressure coefficient. Full-scale simulations for PPTC Case 1.

Next, we investigate the sensitivity of the simulations with respect to the time-step. The DDES of the unsteady cavitation (PPTC Case 2) is considered both in model and full scale. We conduct the analyses using the medium grids. In the analyses, we use the time-steps that correspond to 0.5, 1.0, and 2.0 degrees of propeller rotation. Additionally, we compare the DDES with URANS simulations using the SST $k-\omega$ in both model- and full-scale conditions, where the time-step corresponding to 0.50 degrees of propeller rotation is used. Note that the time step is relative large for the DDES simulation. In all of the results depicted here, we first simulated the case with the URANS SST for one propeller revolution with $\Delta t \widehat{=} 0.50^{\circ}$, after which we started the DDES and switched to the other time steps. The time histories of the thrust and torque coefficients are shown in Figures 19 and 20. The quantities $\Delta K_{T}$ and $\Delta K_{Q}$ denote the ratio of the computed value and the corresponding measured model-scale value, that is, $\Delta K_{T}=K_{T, C F D} / K_{T, E F D}$, for instance. The thrust coefficients in the frequency domain are shown in Figure 21. The average value of the model-scale $K_{T}$ with DDES does not show great dependency on the time step. The URANS SST gives a lower thrust coefficient than the DDES in model scale. The average $K_{Q}$ shows more variation with respect to the utilized time step. The average value of the full-scale torque coefficient seems to reduce as the time is reduced. More frequency content 
in the global forces is seen as the time increment is reduced. Especially in full-scale conditions, the difference between the smallest time step to the other simulations is relatively large, and the URANS SST yield rather similar results to the DDES with twice the time-step. The DDES with $\Delta t \widehat{=} 2.00^{\circ}$ does not yield comparable frequency content in the time histories.
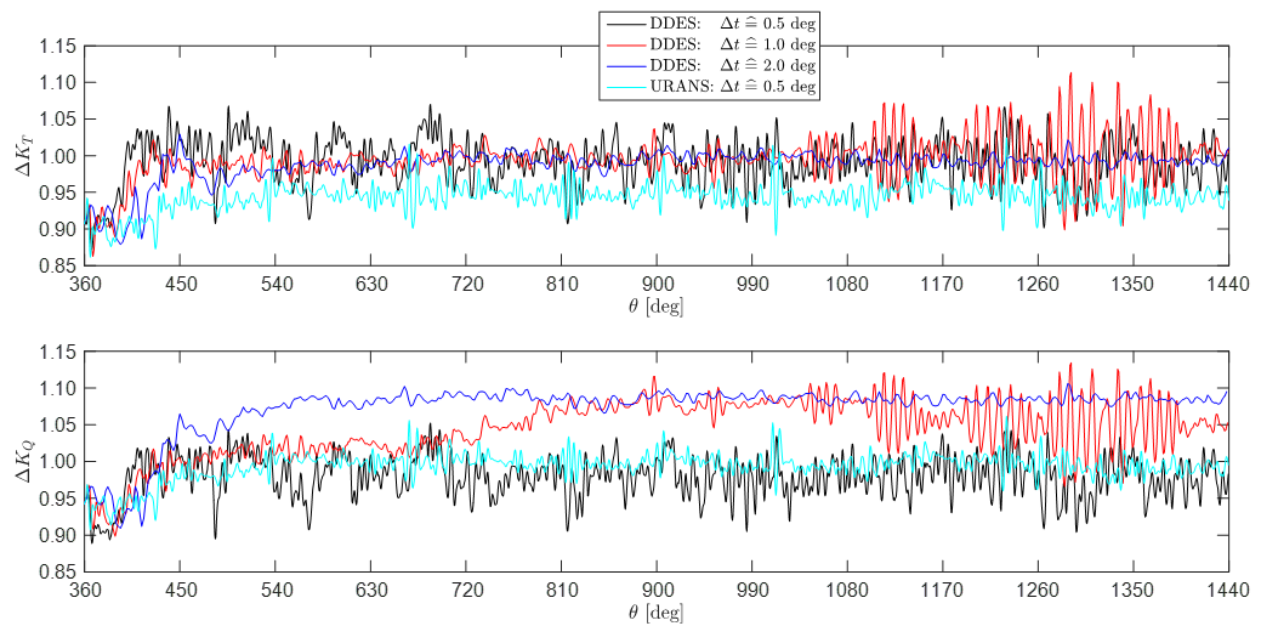

Figure 19. Time histories of the propeller thrust coefficient for PPTC Case 2. Model-scale DDES and URANS simulations with different time-steps.
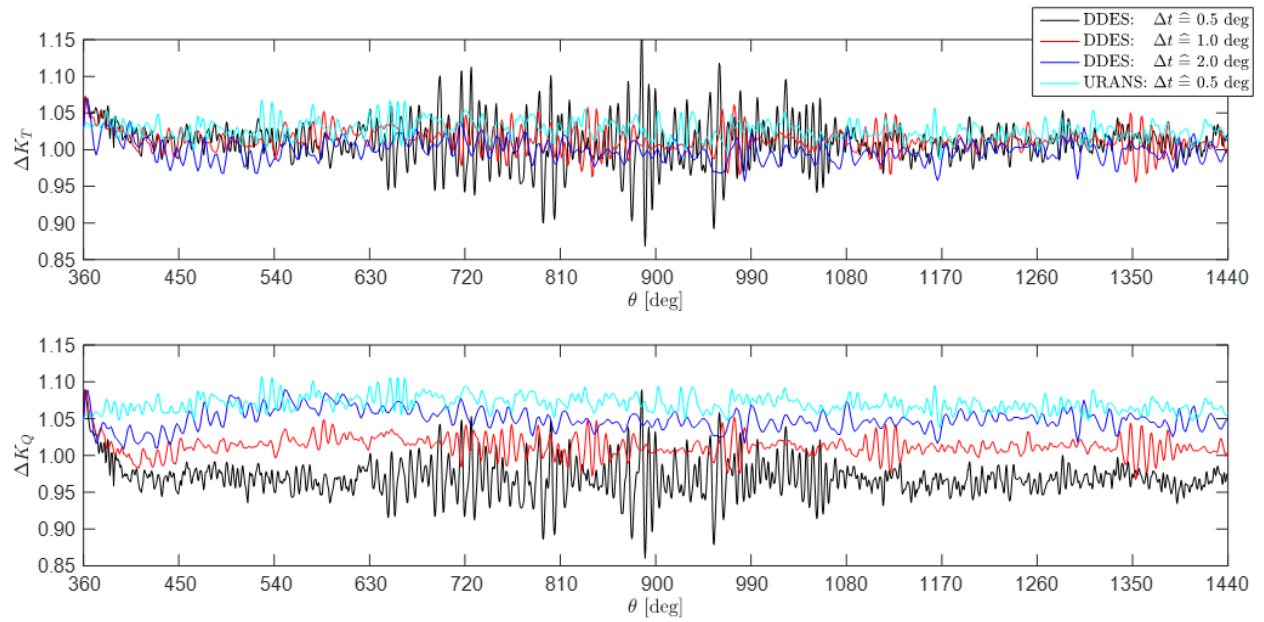

Figure 20. Time histories of the propeller thrust coefficient for PPTC Case 2. Full-scale DDES and URANS simulations with different time-steps.

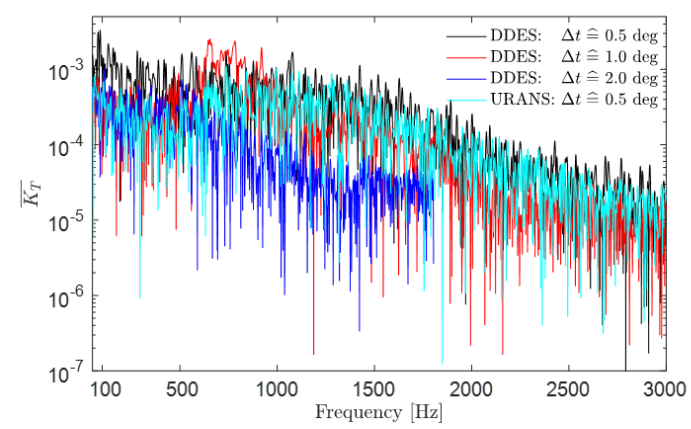

(a) Model-scale.

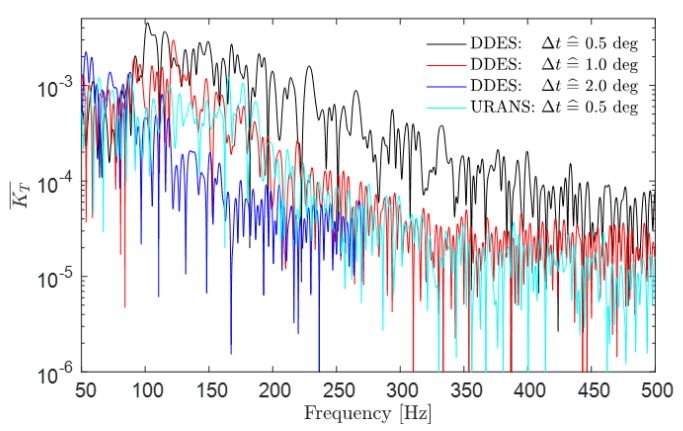

(b) Full-scale.

Figure 21. Propeller thrust coefficient in the frequency domain for PPTC Case 2. 


\section{Model- and Full-Scale Propeller Cavitation}

\subsection{Cavitation Patterns for PPTC Propeller, Case 1 Conditions}

We compare the cavitation patterns in model- and full-scale for the PPTC Case 1. Cavitation close to the blades is visualized in Figure 22, and the overall cavitation patterns are shown in Figure 23. The near-blade cavitation patterns, that is, the root cavitation as well as the LE sheet cavitation transforming into tip vortex cavitation, are very similar between the different simulation conditions. We observe little difference in the model-scale simulations using the SST or TM at the investigated Reynolds numbers. The root cavitation area appears larger in the full-scale conditions, extending past the trailing edge of the blades. The model-scale simulations at $R e_{1}$ yield a slightly narrower tip vortex cavitation, especially farther downstream from the propeller. The tip and hub vortex shape and extent were well reproduced also in full-scale conditions, although the extent of the tip vortex cavitation is slightly less in full scale.

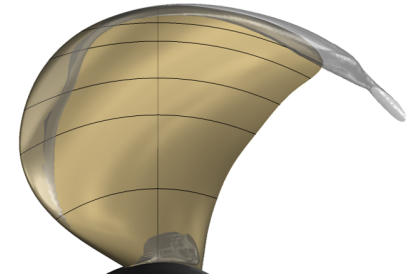

(a) Model scale, SST, $R e_{1}$.

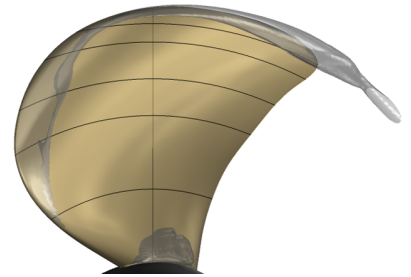

(b) Model scale, TM, $R e_{1}$.

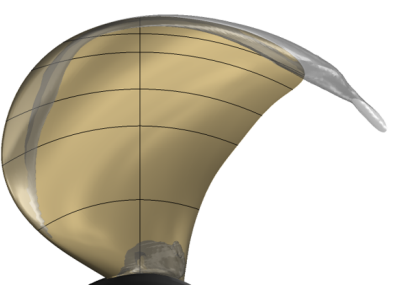

(c) Model scale, SST, $R e_{2}$.

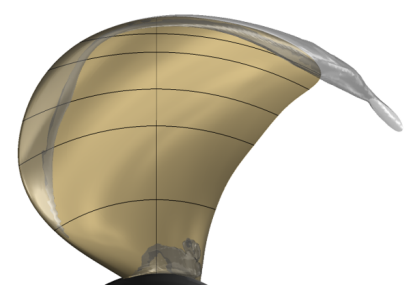

(d) Model scale, TM, $R e_{2}$.

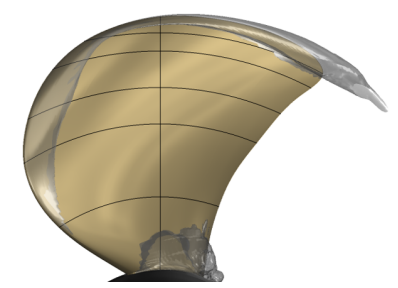

(e) Full scale, SST.

Figure 22. Comparison of the predicted near-blade cavitation observation for the PPTC Case 1 in model- and full-scale conditions.

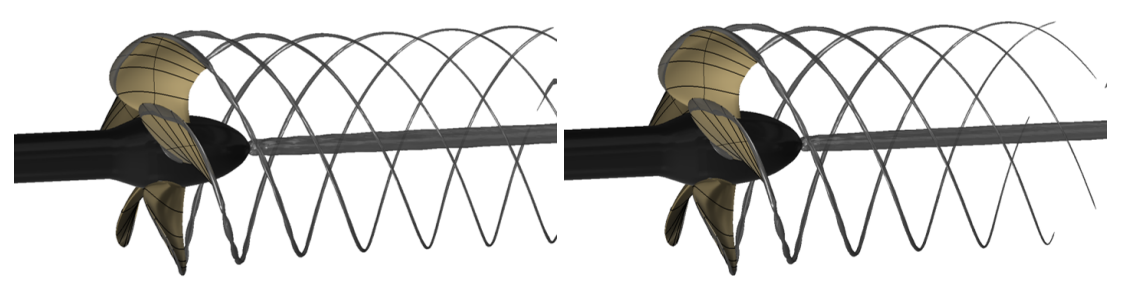

(a) Model scale, SST, $R e_{1}$.

(b) Model scale, TM, $R e_{1}$.

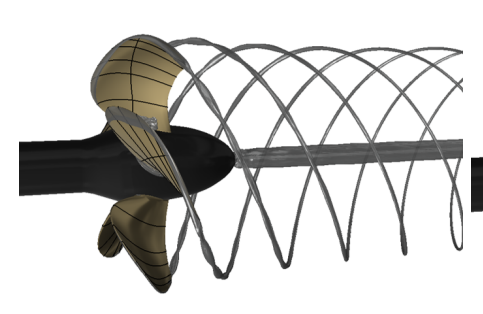

(c) Model scale, SST, $\operatorname{Re}_{2}$.

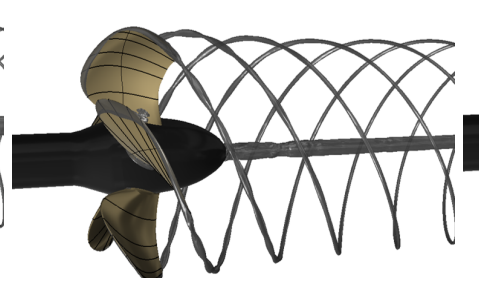

(d) Model scale, TM, $R e_{2}$.

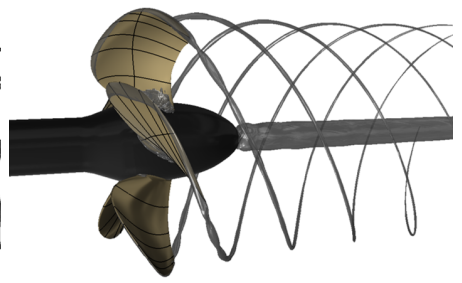

(e) Full scale, SST.

Figure 23. Comparison of the predicted cavitation observation for the PPTC Case 1 in model- and full-scale conditions. 
Next, we investigate the rate of evaporation within the cavity volume in for the PPTC propeller in Case 1 conditions. Figure 24 shows the results on the blade, and Figure 25 with a closer look near the LE. Here, the rate of evaporation is visualized at various cuts in the cavity volume in the axial direction. Also the surface flow on the blades are shown. The boundary layer flow is also discussed in Section 6 . The figure depicts both the model- and full-scale results for the propeller, and the model-scale results are those obtained with the SST and the transition models. The rate of evaporation in model scale is strong at the core of the tip vortex, whereas condensation occurs at the outer edge of the cavitating vortex. Similar trends are also present in full-scale, although the rate is less. A more complex interplay of the evaporation and condensation takes place on the sheet cavities close to the blades: the LE and root areas. The sheet cavity closure line near the leading edge, and corresponding transition between the evaporation and condensation, shifts toward a greater chord length from the leading edge in full-scale conditions. This is quite clearly evidenced also by the orientation of the blade surface flow, where the streamlines on the surface are straighter in full scale until the location of the re-entrant jet. The flow within the sheet cavitation near the leading edge is more complex in model scale.

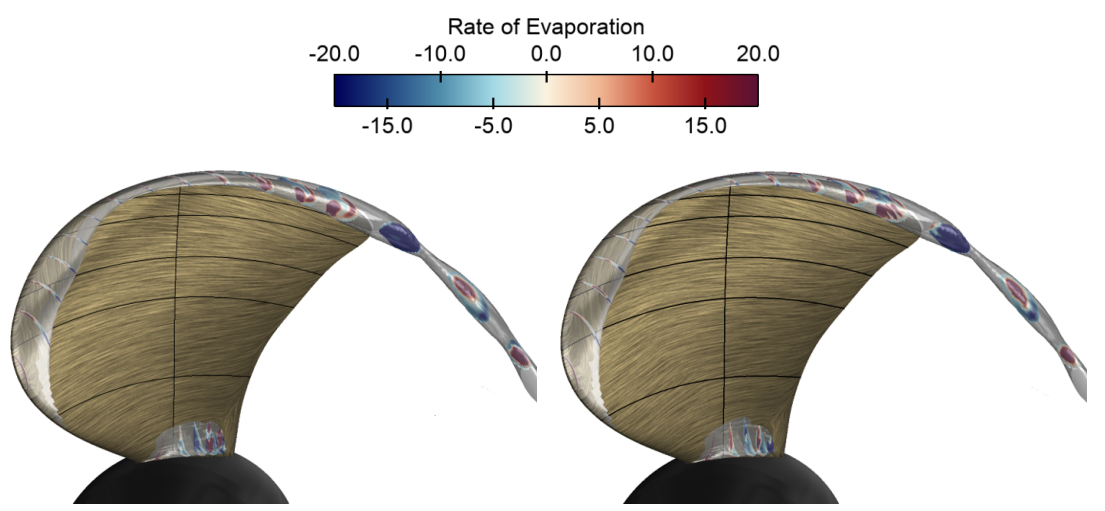

(a) Model scale; $\mathrm{SST}, \mathrm{Re}_{1}$.

(b) Model scale; $\mathrm{SST}, \mathrm{Re}_{1}$.

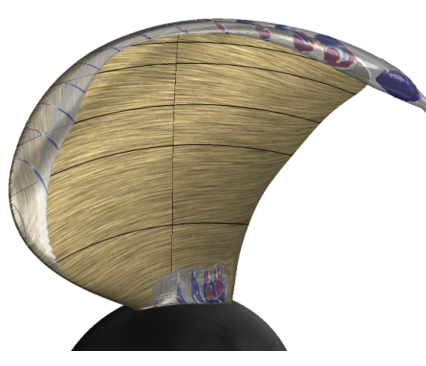

(c) Model scale; SST, $\mathrm{Re}_{2}$.

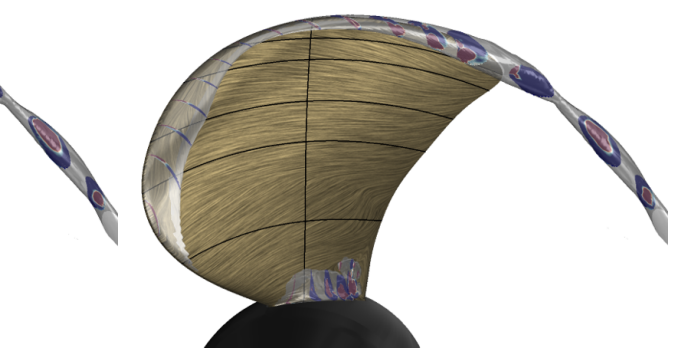

(d) Model scale; TM, $R e_{2}$.

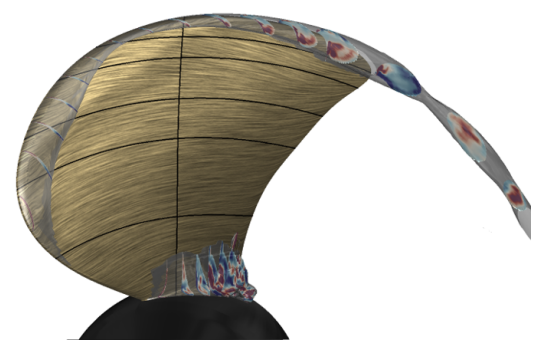

(e) Full scale; SST.

Figure 24. Rate of evaporation $\left[\mathrm{kg} / \mathrm{m}^{3} \mathrm{~s}\right]$ within the cavity volume in model and full scale for the PPTC Case 1. 


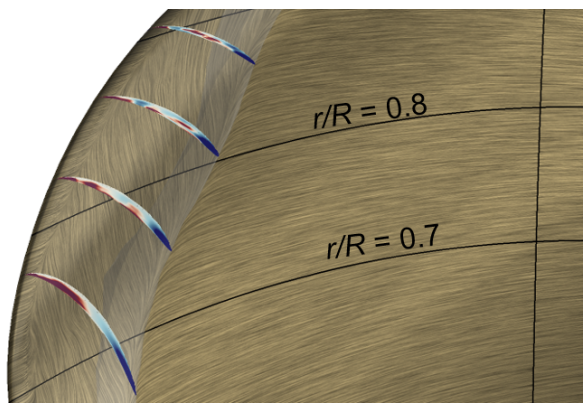

(a) Model scale; SST, $\operatorname{Re}_{1}$.

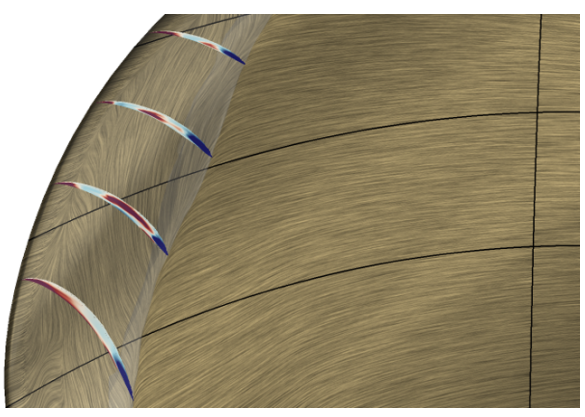

(c) Model scale; TM, $R e_{1}$.

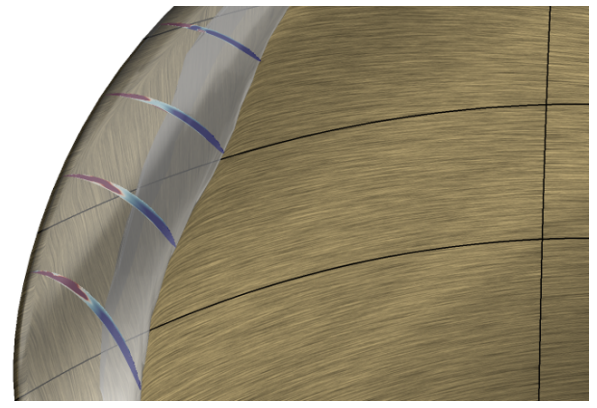

(b) Model scale; SST, $R e_{2}$.

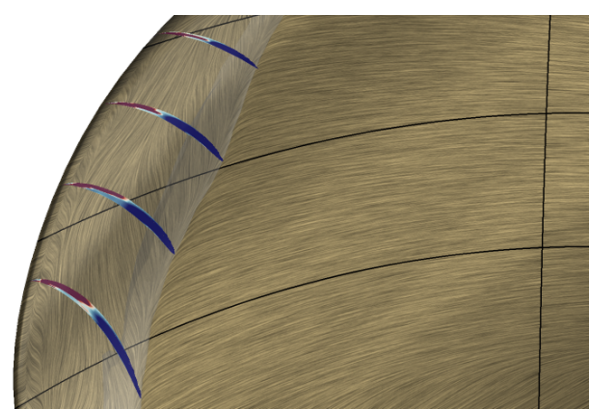

(d) Model scale; TM, $R e_{2}$.

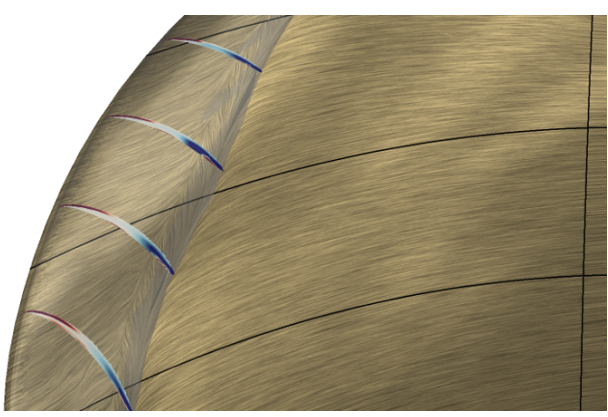

(e) Full scale; SST.

Figure 25. Rate of evaporation $\left[\mathrm{kg} / \mathrm{m}^{3} \mathrm{~s}\right]$ within the cavity volume in model and full scale for the PPTC Case 1. Closer view near the LE. The color scale is the same as in Figure 24.

We note some differences in the sheet cavitation structure in the model-scale conditions, depending on the Re and the utilized turbulence model. The surface flow is directed almost straight upwards toward the tip with SST at both investigated Reynolds numbers, and more waviness is seen at lower Re. The chordwise location of the line dividing the regions of transition from the circular to upward surface flow just after the leading edge shifts closer to the LE at the higher Re (similar shift is seen with TM). At model-scale $R e_{2}$, the SST and TM show clear twofold behavior: strong evaporation close to the LE and condensation after approximately half of the length of the cavity. We observe that condensation occurs within a fine layer just close to the blade, under the region of the strong evaporation. At model-scale $R e_{1}$, we see a more mixed behavior with two distinct regions of evaporation and condensation.

\subsection{Cavitation Patterns for PPTC Propeller, Case 2 Conditions}

Next, in Figure 26 we compare the cavitation patterns close to the blades and in the slipstream of the propeller in model- and full scale for the PPTC Case 2. Furthermore, the cavitation behind the propellers and on the pressure sides of the blades is shown in Figure 27. The near-blade cavitation pattern is again similar in model- and full-scale conditions. The fine sheet cavity covers a large part of the propeller, as it extends from the root to the tip. The inception of the tip vortex cavitation 
appears similar between the simulations. Unsteady shedding of the root cavitation occurs in both simulations, subsequently transforming into cloudy cavitation in the propeller wake. The full-scale DDES predict clearer cavitation structures just behind the blades, whereas the model-scale DDES shows more extensive cavitation farther downstream of the propeller. We continue with the analyses of the unsteady cavitation shedding in Figure 28.

In Figure 27, we note that the propellers exhibit a sheet cavitation on the pressure side, originating from the root and extending up until the tip of the blade. As it approaches the tip, the pressure side sheet cavitation turns very fine, and ultimately joins the suction side at the propeller tip to form the tip vortex cavitation. This is also visible in Figure 26a,b. Although not shown here, we note that this extension of the fine pressure side sheet cavitation was not so clearly visible in the results obtained with the RANS models utilized. The propellers also show root cavitation on the pressure sides of the blades. A range of cavitation structures of varying sizes appear in the propeller wake, as we also noted earlier in Figures 9 and 26. Again, we observe clearer these cavitation structures just behind of the full-scale propeller, whereas in model-scale conditions, they appear farther downstream.

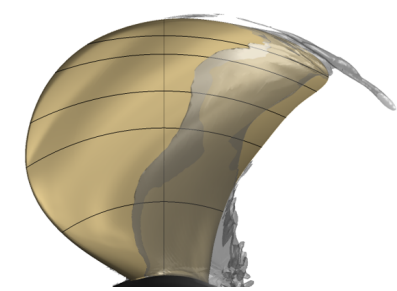

(a) Model scale.

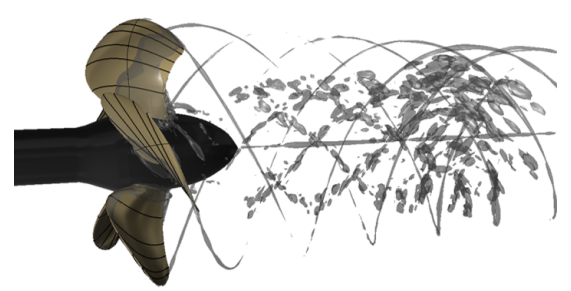

(c) Model scale.

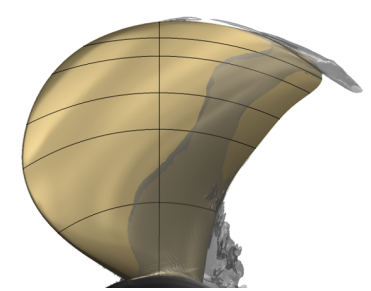

(b) Full scale.

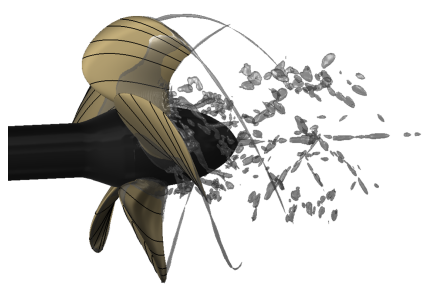

(d) Full scale.

Figure 26. Comparison of the predicted cavitation patterns for the PPTC Case 2 in model- and full-scale conditions. Both results are from DDES.

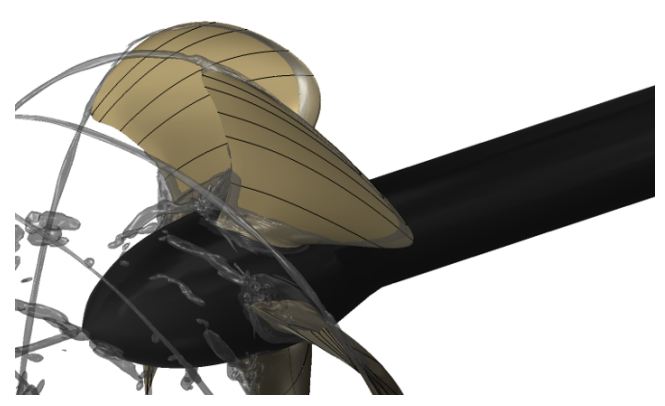

(a) Model scale.

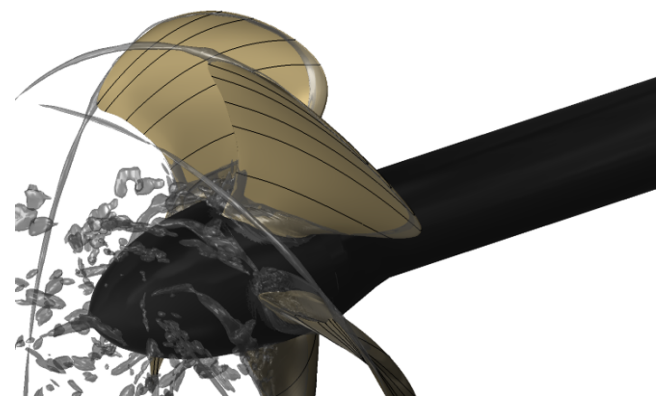

(b) Full scale.

Figure 27. Comparison of the predicted cavitation patterns behind the propeller and on the pressure side of the blades for the PPTC Case 2 in model- and full-scale conditions. Both results are from DDES. 

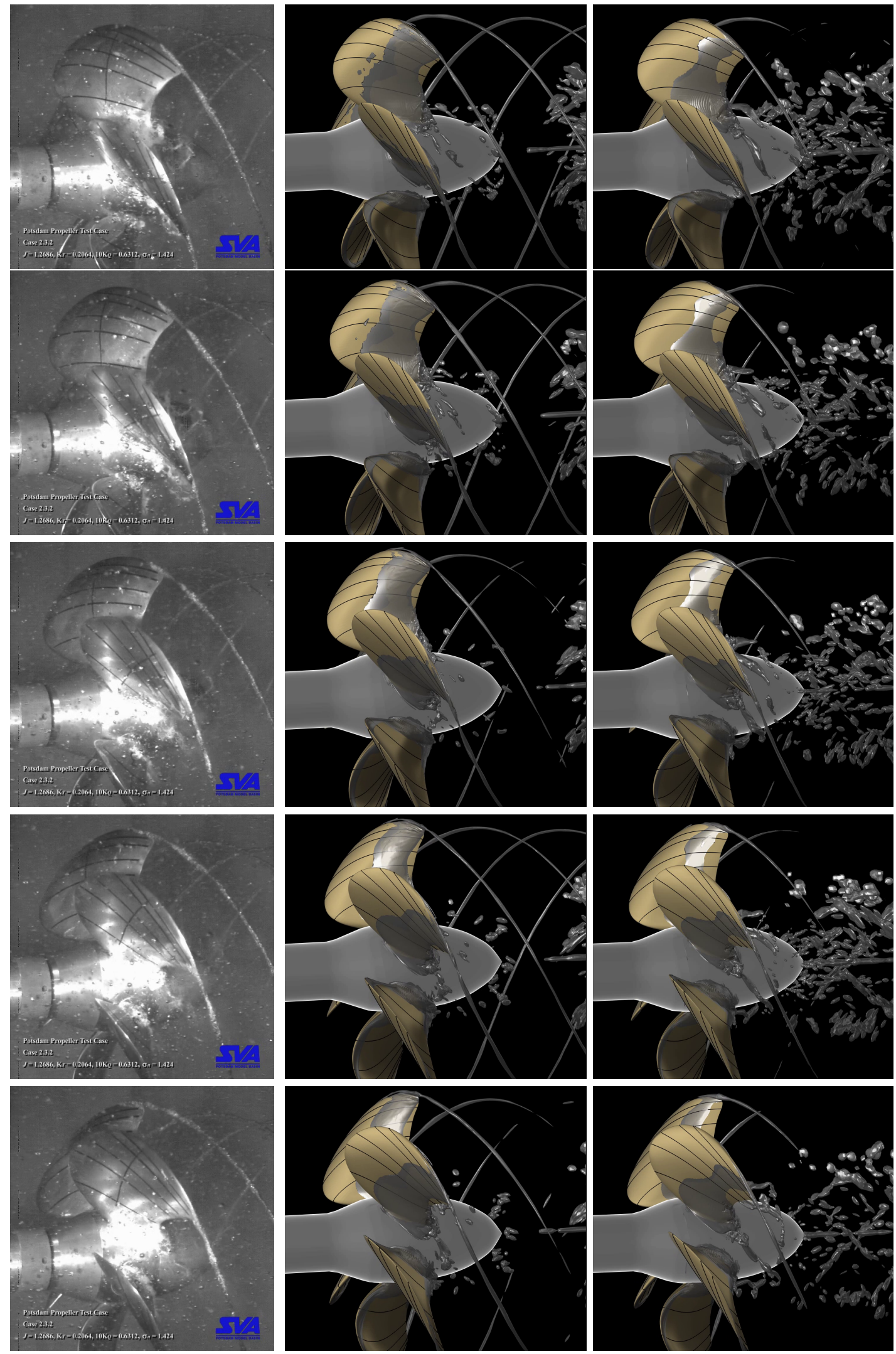

Figure 28. Cavitation growth and detachment in model- and full-scale PPTC Case 2 conditions. Left column: Experiments (model scale), reproduced with permission from Lars Lübke (SVA). Middle column: DDES Model scale. Right column: DDES Full scale. The time increment between each frame corresponds to 10 degrees of propeller rotation. 
To visualize the model- and full-scale cavitation dynamics for the PPTC Case 2 conditions, we show a series of snapshots of the DDES for this case in Figure 28. In the figure, we view the simulated cavitation growth and detachment in model- and full-scale conditions together with the still frames from the video recording of the cavitation tunnel experiments (https:// player.vimeo. $\mathrm{com} /$ video/159048901). We show five frames in the figure, and the time increment between each frame corresponds to 10 degrees of propeller rotation, corresponding to $\Delta t=1.389 \mathrm{~ms}$ in model scale and $9.259 \mathrm{~ms}$ in full scale. The propeller has root cavitation both on the suction and pressure sides of the blades. From these, unsteady vapor structures are shed to the wake. Particularly close to the hub surface, these appear as relatively long filaments. Comparing the model- and full-scale conditions, the length of these filaments is greater in full scale. The full-scale DDES show more extensive cavitation structures just behind the blades. Additionally, the vapor structures in the "cloud cavitation" region in propeller wake were more dynamic in the full-scale simulations, that is, they exhibited a relatively higher frequency "bursting" behavior. These structures take place also closer to the propeller in the full-scale conditions, cf. Figures 9 and 26. We observed a similar behavior when comparing the unsteady cavitation characteristics in model- and full-scale SST simulations, although these are not shown here. The tip vortex cavitation has some instability in the DDES: its extent and shape featured rapid changes occasionally in the simulations. This behavior was likely caused by the bursting of the slipstream "cloudy" cavitation, and the subsequent pressure waves caused by the collapse. In the experiments, some instability in the tip vortex cavitation is also seen, although similar appearance/disappearance was not observed. The shape or the extent of the fine sheet cavitation on the blades, upward of approximately $r / R \approx 0.5$, does not appear very transient in either simulation; occasional appearance of bubbly cavitation is visible in the experiments.

\subsection{Cavitation Patterns and Evaporation Rate for TLP Propeller}

We compare the cavitation patterns of the TLP propeller in the model- and full-scale conditions. Figure 29 shows both the cavitation close to the blades and the overall cavitation patterns. The results obtained with the SST model are shown. The cavitation patterns on the blades mostly resemble each other in model- and full-scale conditions. The model-scale cavitation detaches from the blade before the trailing edge, and the sheet cavitation covers the entire blade surface in full scale. In the full-scale conditions, this cavity detachment is different, as it remains attached to the blade until the trailing edge. The sheet cavitation transforms into vortex cavitation before the tip of the blade in both cases. Cavitation of the secondary tip vortex due to the endplate is visible in both simulations, although the very fine vortex cavitation does not extend very far in either case. The tip vortex shape and extent in the full-scale conditions is similar to the model-scale observations, although the extent of the tip vortex cavitation is less in full scale.

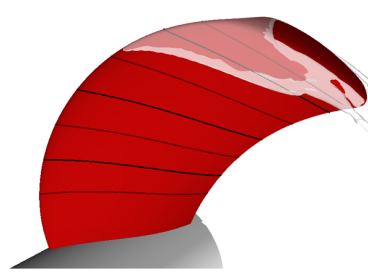

(a) Model scale.

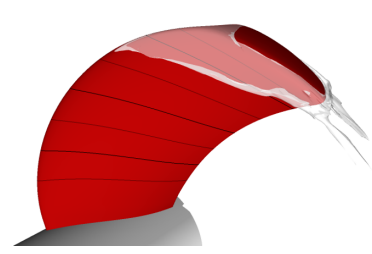

(b) Full scale.

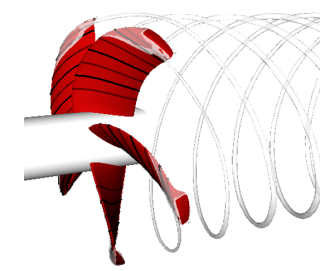

(c) Model scale.

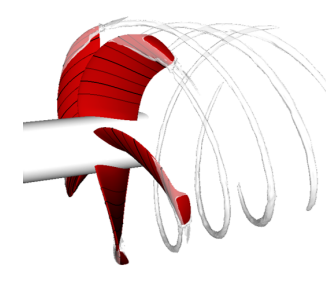

(d) Full scale.

Figure 29. Comparison of the predicted cavitation patterns for the TLP in model- and full-scale conditions. Both results are from SST simulations.

We study the rate of evaporation within the cavity volume for the TLP propeller in Figure 30. Similarly to the above, the rate of evaporation is visualized at various cuts in the cavity volume in the axial direction. Also the surface-restricted streamlines on the blades are shown. The figure shows both the model- and full-scale results for the propeller, and the model-scale results are those obtained 
with the SST and the transition models. The boundary layer flow is further discussed in Section 6 . The rate of evaporation in model scale is stronger for the TLP at the core of the tip vortex, compared to the PPTC case. The TLP propeller has a little lower $J$ value, and the cavitating tip vortex is finer. Condensation occurs at the outer edge of the cavitating vortex. These trends are also present in full scale, and again the rate of evaporation is less. The sheet cavity on the blade surface is quite thick. Evaporation seems to dominate closer to the leading edge, whereas more condensation is seen closer to the mid-chord position. The surface-restricted streamlines are straighter in full scale, also underneath the sheet cavity. The flow within the sheet cavitation is more complex in model scale, which is visible in both the SST and transition simulations. The model-scale simulations with the SST and transition model yield relatively similar results.

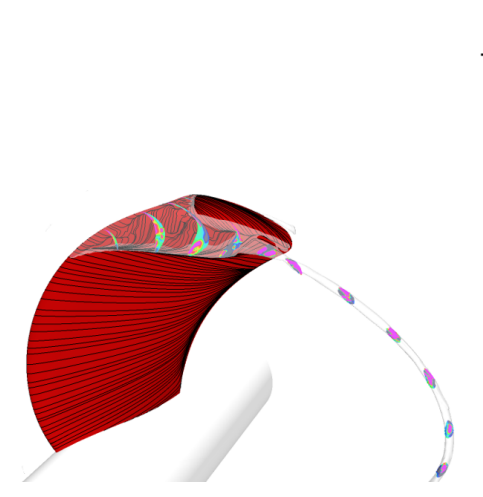

(a) Model scale; SST.
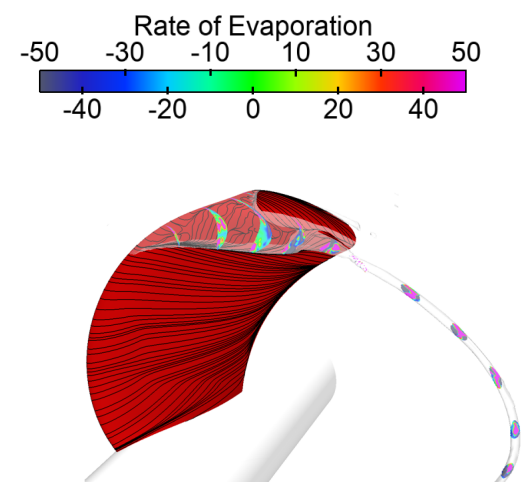

(b) Model scale; TM.

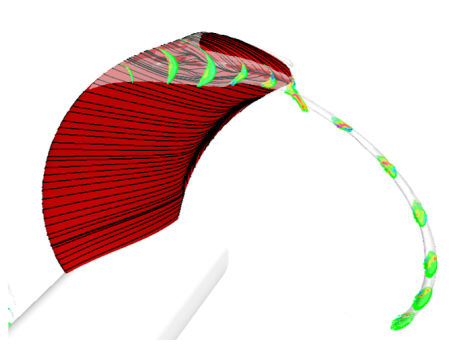

(c) Full scale; SST.

Figure 30. Rate of evaporation $\left[\mathrm{kg} / \mathrm{m}^{3} \mathrm{~s}\right]$ within the cavity volume in model and full scale for the TLP propeller. Perspective views near one blade. Cavitation is shown as the grey transparent surface, and the surface-restricted streamlines on the blades as the black curves.

\section{Near-Blade Flow: Skin-Friction and Pressure Distributions}

\subsection{Near-Blade Flow Analysis for PPTC}

We show a comparison of the skin-friction and pressure coefficients in Figures 31 and 32 in modeland full-scale conditions. The distributions are compared on the radius $r / R=0.7$ for PPTC Case 1 . The vertical scale is limited in these graphs to better visualize the results, and the peak skin-friction and pressure coefficient values at the LE are not shown. The highest values were produced in the SST and TM simulations with $R e_{1}$, while the lowest in the full-scale conditions. The $C_{p}$ values at the leading edge are nearly identical in all simulations.

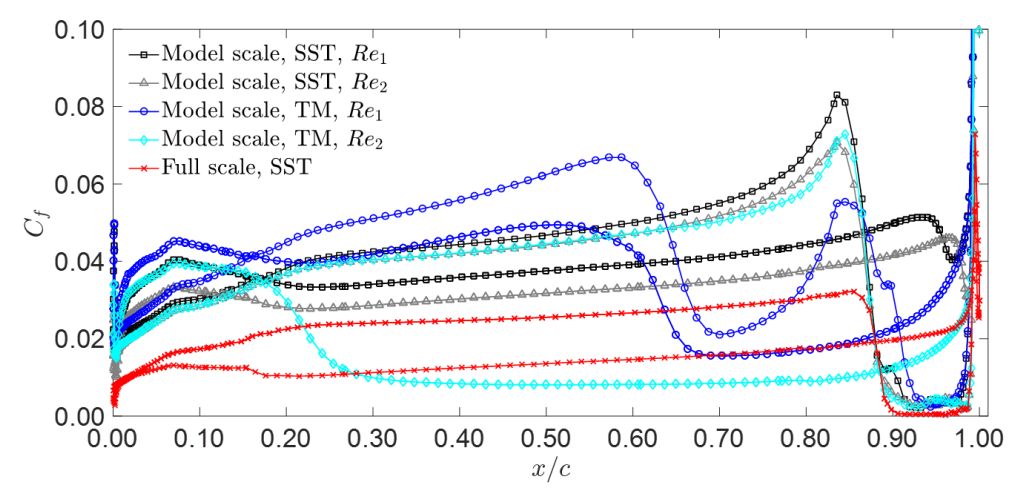

Figure 31. Comparison of skin-friction coefficients at the radius $r / R=0.7$ on the blades. PPTC Case 1 conditions in model- and full-scale. 

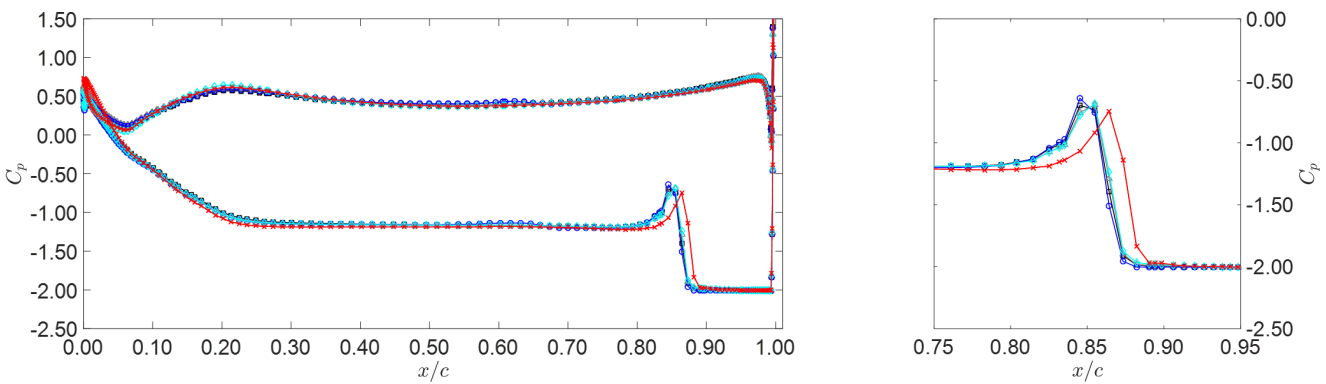

Figure 32. Comparison of pressure coefficient at the radius $r / R=0.7$ on the blades. PPTC Case 1 conditions in model- and full-scale. The legend is the same as in Figure 31.

Figure 33 shows the skin-friction and pressure coefficients on the blades in model- and full-scale conditions. For reference, the same radii as before are shown as the black circles drawn on the blades. The boundary layer flow was mostly circumferentially directed along the blade for the model- and full-scale SST simulations. Flow separation takes place at the root cavitation close to the TE in all simulations. The SST model predicts fully turbulent flow on the wetted parts in model scale, and little difference in the surface flow is seen between the two model-scale SST simulations. A clear difference in the surface streamlines for the PPTC in Figures 24, 25 and 33 is seen in the areas covered by the sheet cavitation. The model scale simulations using the SST and TM show a main flow direction under the sheet cavity before the cavity closure line that is more wavy and radially directed, whereas the surface streamlines are more organized and circumferentially oriented in full scale. The TM at higher $R e$ predicts a laminar flow region below $r / R \approx 0.6$, and the TM with lower Re shows laminar flow zones extending slightly above $r / R \approx 0.6$, but transitioning to turbulent flow before the mid-chord. The effect of cavitation on the surface restricted streamlines is significant. The re-entrant jets in both model- and full-scale simulations are directed towards the cavitating tip vortex at the closure line of the sheet cavitation as observed before in model-scale conditions [10,24].

Generally, the skin-friction coefficient drops to very small values in the areas underneath the cavitation and attains greater values in the wetted parts of the blades. The full-scale skin-friction coefficient values are mostly below those seen in model-scale. We observe a peak in the $C_{f}$ immediately after the cavity closure in all model-scale simulations; this is not visible in full-scale conditions. The skin-friction coefficient under the LE sheet cavity appears as more uniform in the model-scale SST and TM simulations with the higher $R e_{2}$, when compared to the results with the lower Reynolds number. The $C_{f}$ exhibits some deviations between the model-scale SST simulations, obtaining greater values at smaller radial locations with the higher Reynolds number. At $r / R=0.7$, the simulations with higher $R e$ produces greater $C_{f}$ magnitude. The TM simulations with the lower Re predicts laminar flow regions below $r / R=0.7$, visible in the surface flow orientation and as a lower $C_{f}$. The TM simulations with $R e_{2}$ predicts a laminar flow zone roughly below $r / R \approx 0.6$, extending almost the entire chord length. Exceptions are seen in the root area, where flow separation takes place due to the cavitation. The skin-friction distributions between the TM simulations at the investigated Re are quite different.

The TM at $R e_{1}$ shows a peculiar chordwise development of the $C_{f}$ : small values under the LE sheet cavitation rebound to higher values after the closure line (corresponding to the other simulations), again attaining small values in the laminar flow zone, after which flow transitions to a turbulent region, the skin-friction coefficient increases and the surface flow changes to a more circumferential orientation. A similar development is seen on the pressure side, but without the low $C_{f}$ due to lack of cavitation there: laminar flow region extends to $x / c \approx 0.65$, after which the skin-friction starts to increase, and the surface flow (not shown here) transforms from nearly radial to more circumferential orientation. The TM at the higher Reynolds number predicts a skin-friction distribution in line with the model-scale SST simulations above approximately $r / R=0.6$ on the suction side, albeit with slightly smaller values for the $C_{f}$. On the blade pressure side, however, the TM simulations at the higher Reynolds number show strange development. After the LE peak in the $C_{f}$, the model predicts laminar flow with a nearly constant skin-friction coefficient. The coefficient begins to increase only once we have reached a 
chordwise position of $x / c \approx 0.3$. Inspection of the surface flow on the pressure side revealed similar observations. The reason for this is not clear, and further studies should be carried out in the future.

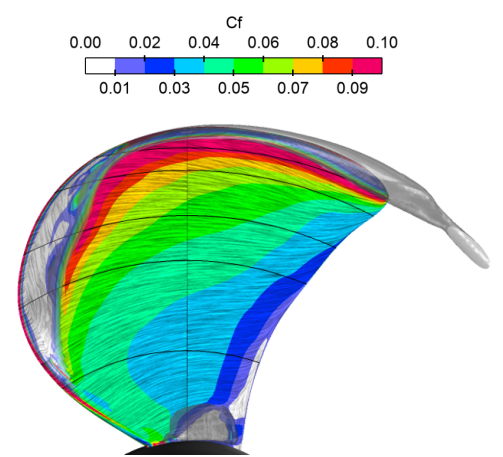

(a) Model scale, SST, $R e_{1}$.

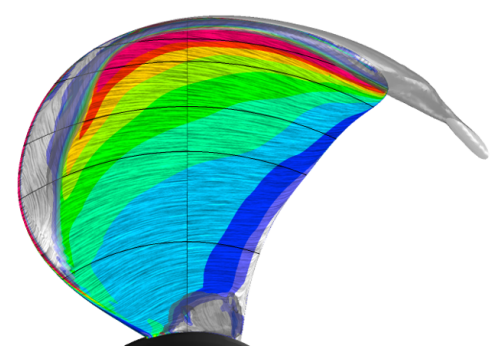

(b) Model scale, SST, $R e_{2}$.

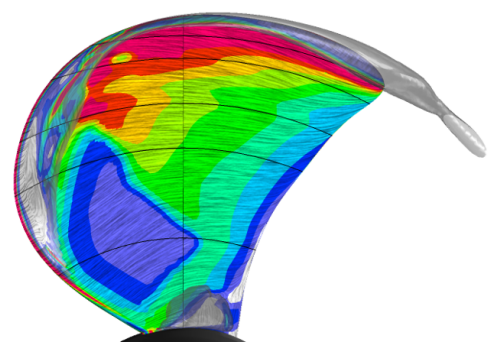

(c) Model scale, TM, $R e_{1}$.

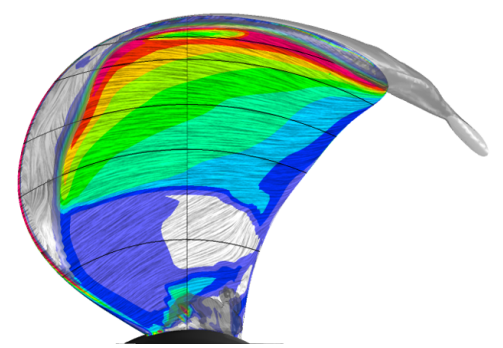

(d) Model scale, TM, $R e_{2}$.

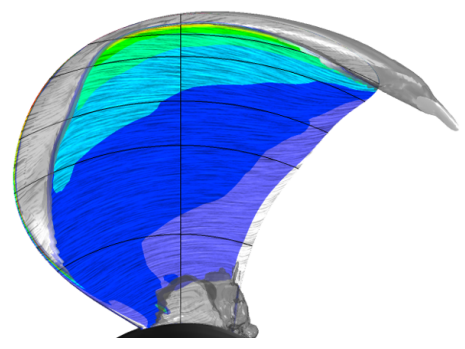

(e) Full scale, SST.

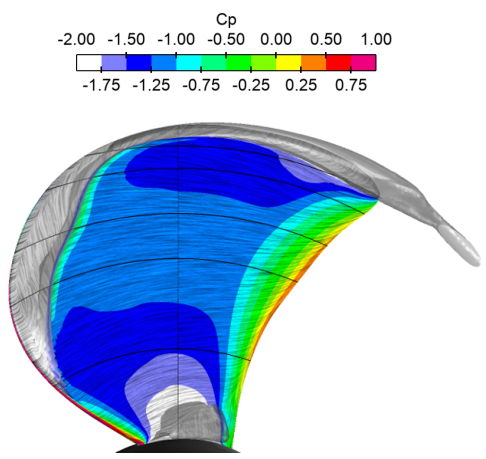

(f) Model scale, SST, $R e_{1}$.

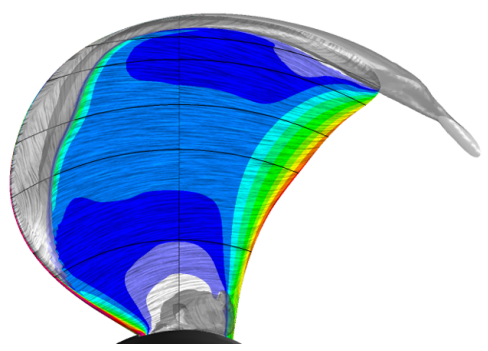

(g) Model scale, $\mathrm{SST}, \mathrm{Re}_{2}$.

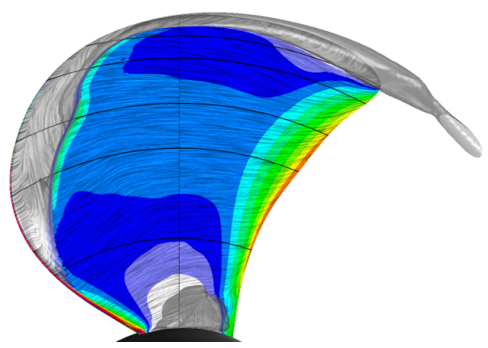

(h) Model scale, TM, $R e_{1}$.

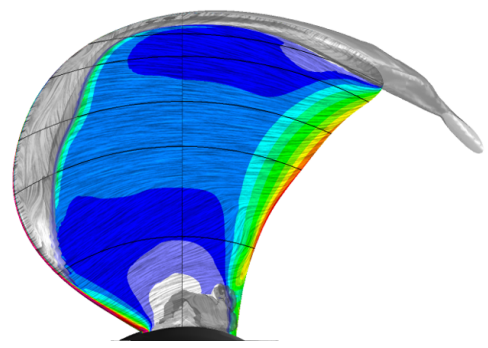

(i) Model scale, TM, $R e_{2}$.

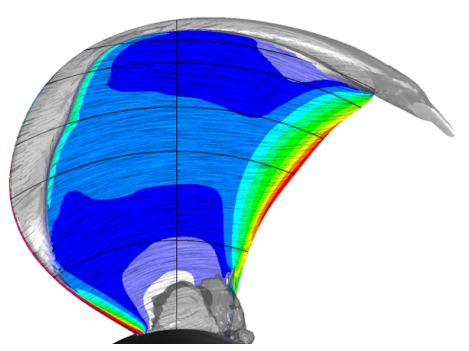

(j) Full scale, SST.

Figure 33. Comparison of near-blade skin-friction and pressure coefficients for PPTC Case 1. Left column: $C_{f}$. Right column: $C_{p}$. 
The overall blade pressure distributions are very similar in the model-scale SST and TM simulations, and little sensitivity is seen with respect to the Reynolds number. The low pressure region at the middle of the blade is slightly narrower in full-scale conditions (Figure 33j). Also, a higher surface pressure in the tip area, below the cavitation zone, is seen for the full-scale conditions. The root cavitation extent is larger in full scale, and the low pressure region near the root covers a larger area in full scale as well. The pressure coefficient distributions at the $r / R=0.7$ radius, shown in Figure 32, are very similar between all simulations, except for the TE region and the location of the cavity closure. The surface pressure is limited to the vapor pressure between the LE and $x / c \approx 0.85$, after which it increases rapidly. This increase takes place sooner and a bit sharper in full-scale conditions, whereas the model scale result appears as quite insensitive of the Reynolds number or the used turbulence models. The strength of the pressure increase, peaking at a value bit below -0.5 , is similar between all simulations.

\subsection{Near-Blade Flow Analysis for TLP}

The skin-friction and pressure distributions on the blades of the TLP are shown in Figures 34 and 35, where we compare the model- and full-scale conditions. For reference, the same radii as before are shown as the black circles drawn on the blades.

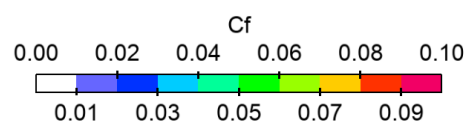

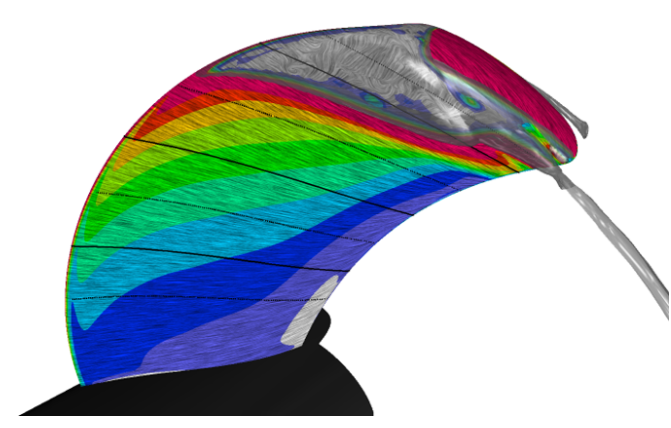

(a) Model scale, SST.

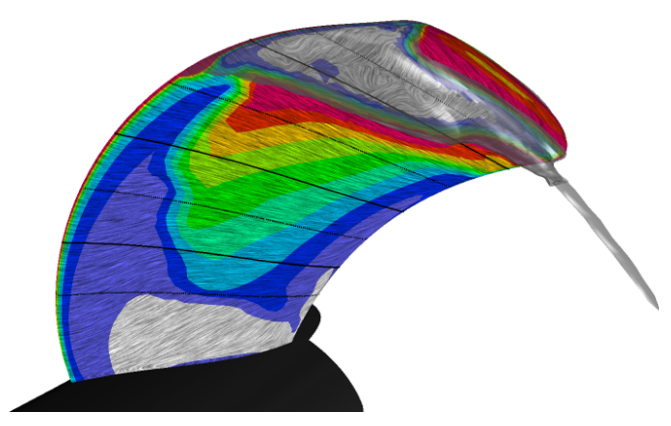

(b) Model scale, TM.

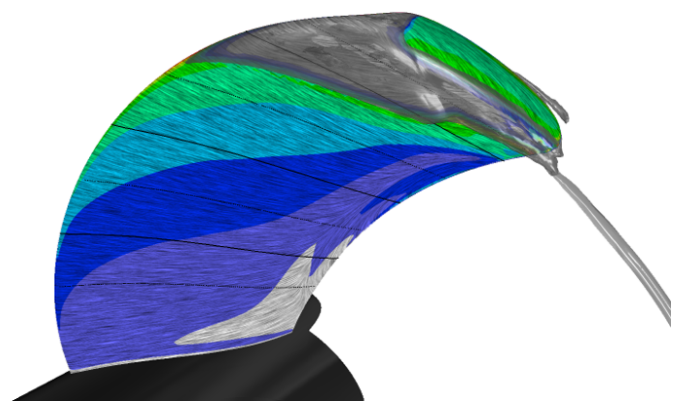

(c) Full scale, SST.

Figure 34. Comparison of near-blade skin-friction for TLP. 


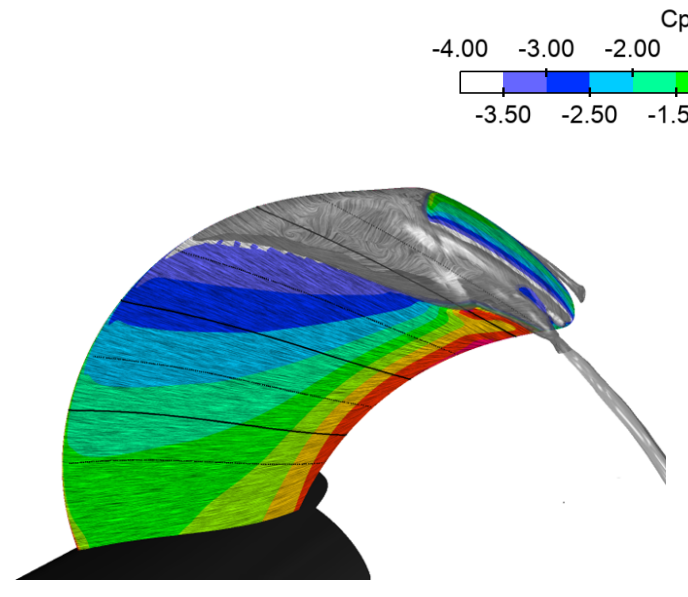

(a) Model scale, SST.

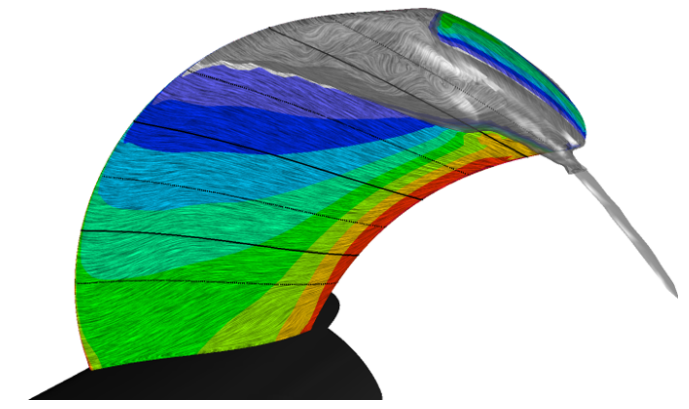

(b) Model scale, TM.

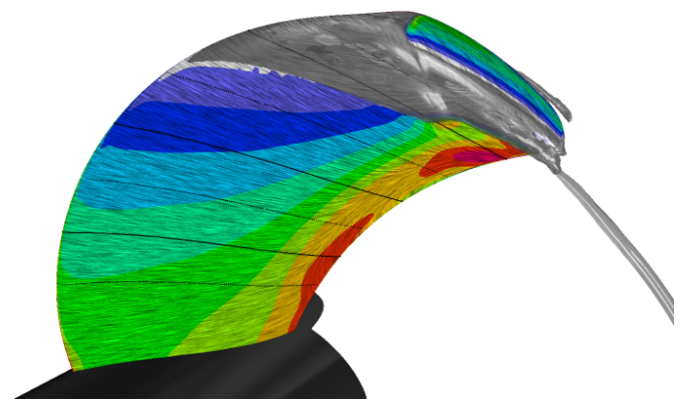

(c) Full scale, SST.

Figure 35. Comparison of blade pressure coefficient for TLP.

The boundary layer flow for TLP is mostly circumferentially directed along the blade for the model- and full-scale simulations. The surface flow turns upward before the TE in full-scale conditions, which appears also in the $C_{f}$ distribution as lower values. Otherwise, the shapes of the $C_{f}$ distributions between the two SST simulations are quite alike, albeit the full-scale conditions yield less chordwise variation and overall lower values for the skin-friction coefficient. The SST model predicts fully turbulent flow on the wetted parts in model scale, and little difference in the surface flow is seen between the SST simulations. The TM predicts laminar flow regions near the LE and close to the root below $r / R=0.4$; this is also visible as the zones of smaller values in the skin-friction distributions when compared to the model-scale SST. The laminar flow regions predicted by the TM do not have a visible influence on the $C_{p}$. The TM predicts a little lower skin-friction coefficient on the end plate, although we do not see much differences in the surface flow between the SST and TM in model scale there. A clear difference in the surface streamlines is seen in the areas covered by the sheet cavitation, especially in model-scale conditions. The model-scale simulations show a significantly more wavy and partly radially directed main flow direction under the sheet cavitation. Small spots of flow separation appear, evidenced also as quite complex phase change phenomena (cf. Figure 30). The flow underneath the sheet cavity is more regular and circumferentially oriented in full-scale than in the model-scale simulations. The sheet cavitation turns narrower but remains attached until the $\mathrm{TE}$, which is also visible as the region of lower $C_{f}$ values that extend to the trailing edge. We do not, however, see a similar effect in the $C_{p}$, which remains low on the blade surface throughout the chord. The skin-friction coefficient underneath the cavitation attains lower values compared to the wetted parts of the blade. The blade pressure distributions are mainly very similar between the model- and full-scale simulations. The different surface flow behavior underneath the sheet cavitation has little effect in the blade pressure distributions which are limited by the vapor pressure. The pressure is 
slightly smaller in full scale near the detachment of the sheet cavity, and then grows to larger values near the trailing edge.

\section{Discussion and Conclusions}

We have numerically investigated single- and two-phase flows around marine propellers in open-water conditions at different Reynolds number regimes. Two types of marine propellers were considered-a conventional and a tip-loaded one-and both steady and unsteady propeller cavitation were investigated. We carried out the simulations using a homogeneous compressible two-phase flow model with RANS and hybrid RANS/LES turbulence modeling approaches. Transition was accounted for in the model-scale simulations by employing a transition model. The sensitivity of the results to the utilized numerical grid and time step size was studied. We conducted the sensitivity studies of the cavitation simulations with respect to the propeller global performance characteristics and cavitation patterns, as well as to the blade loading in terms of skin-friction and pressure distributions. These investigations were done both in model- and full-scale conditions.

The model-scale propeller performance and cavitation patterns are captured well in the numerical simulations, with generally little difference between the utilized turbulence models in the model-scale conditions. The global performance characteristics agreed favorably with the experimental values, the deviations being no larger than few per cent on the finest numerical grids. In addition, the unsteady hybrid RANS/LES simulations predicted the average global performance that matched closely with the experiments. The model-scale URANS performed scantly worse. Particularly, the TM predicted a large difference with respect to the experiments in the unsteady case: the low-pressure region on the blades was not sufficient for the inception of cavitation there.

The different cavitation types for both propellers were mostly replicated with the numerical simulations. Especially, the tip and hub vortex cavitation shapes and extents were captured well, in both the steady and unsteady cases. Also, the modal shapes of the cavitating tip vortex were present in the simulation results. We noted an exceptional deviation to the experiments with TM in the investigated unsteady case, however, which merits a further assessment.

The full-scale performance characteristics were also compared to the model-scale values: the simulations indicated a slightly increased thrust and a lower torque coefficient in full-scale conditions, both for wetted and cavitating cases. Considering the cavitation observation, we noted corresponding cavitation types between the model- and full-scale conditions. We observed a tendency of increased cavitation extent as the Reynolds number increases. The tip vortex cavitation in quasi-steady simulations was thicker especially near the blades in the full-scale conditions. The hub vortex for the PPTC propeller was clearly thicker in the full-scale simulation. A similar trend revealed when we compared the model-scale results for the PPTC at the different Reynolds numbers. The root cavitation was also more extensive in full scale. The same trend was observed in the tip vortex cavitation for the TLP propeller: the thickness of vortex cavitation was larger in full scale than in model scale. We observed that the phase change phenomena obtained a slower rate in full-scale conditions. At the same time, dissipation of the cavitating tip vortices was greater in the full-scale simulations. In the case of unsteady computations, the tip vortex cavitation extent was also diminished in full scale. The "cloudy" cavitation in the propeller wake was, then again, more extensive in full scale also appearing closer to the propeller. The simulated cavitation detachment was more comprehensive in full scale. The vapor structures in the "cloud cavitation" region in propeller wake were more dynamic in the full-scale simulations exhibiting a relatively higher frequency "bursting" behavior.

The surface flow on the blades differed clearly between the model- and full-scale simulations in the areas covered by the sheet cavitation for both investigated propellers. The near-blade flow underneath the sheet cavity was more regular and circumferentially oriented in full-scale than in the model-scale conditions. The model-scale simulations show a more wavy and radially directed surface flow direction under the sheet cavitation. 
The skin-friction coefficients were lower in the areas covered by the sheet cavitation in the case of steady cavitation simulations. The blade surface pressure is limited by the vapor pressure under the cavities. As expected, the skin-friction coefficient showed a significant grid resolution and Reynolds number dependency. The blade pressure coefficient distribution, then again, did not show as strong sensitivity. The finer grids predicted quite sharp an increase in the chordwise $C_{p}$ after the LE sheet cavity closure, whereas we noted a lot smoother growth with the coarse grids. The overall blade pressure distributions are very similar in the model-scale SST and TM simulations, and little sensitivity is seen with respect to the Reynolds number or the presence of laminar flow regions. The increase in $C_{p}$ at the LE sheet cavity closure appears sharper and takes place closer to the LE in full-scale conditions.

The unsteady cavitation dynamics in the propeller wake for PPTC Case 2 is extremely complex. The simulations yield the void fractions greater than visible in the cavitation tank test recordings. Predictions of the "cloudy" or bubbly cavitation flow appearances with finite grid densities is very difficult using homogeneous mixture models. In this sense, better results could be achieved with more holistic modeling approaches that are able to better account for the phase change physics, such as inhomogeneous or multi-scale flow models. An extended model of the former type is under development by the authors, and we have obtained promising initial results of propeller cavitation using such an approach.

Author Contributions: Conceptualization, V.V., T.S. and A.S.-C.; methodology, V.V.; software, V.V. and T.S.; validation, V.V., T.S. and A.S.-C.; formal analysis, V.V.; investigation, V.V.; resources, V.V. and A.S.-C.; data curation, V.V. and T.S.; writing-original draft preparation, V.V.; writing-review and editing, T.S. and A.S.-C.; visualization, V.V.; supervision, T.S. and A.S.-C.; project administration, A.S.-C.; funding acquisition, A.S.-C. All authors have read and agreed to the published version of the manuscript.

Funding: This research received no external funding.

Acknowledgments: The authors are very grateful to Michael Brown from NSWCCD for providing the experimental results of the TLP propeller in the forms of open-water data and cavitation tunnel results.

Conflicts of Interest: The authors declare no conflict of interest.

\section{Abbreviations}

The following abbreviations are used in this manuscript:

$\begin{array}{ll}\text { CFD } & \text { Computational fluid dynamics } \\ \text { CLT } & \text { Contracted and loaded tip } \\ \text { CPU } & \text { Centra processing unit } \\ \text { DES } & \text { Detached eddy simulation } \\ \text { DDES } & \text { Delayed detached eddy simulation } \\ \text { EARSM } & \text { Explicit algebraic Reynolds stress model } \\ \text { EFD } & \text { Experimental fluid dynamics } \\ \text { LDV } & \text { Laser Doppler velocimetry } \\ \text { LE } & \text { Leading edge } \\ \text { LES } & \text { Large eddy simulation } \\ \text { MUSCL } & \text { Monotonic upstream-centred scheme for conservation laws } \\ \text { PPTC } & \text { Potsdam propeller test case } \\ \text { RANS } & \text { Reynolds averaged Navier-Stokes } \\ \text { SST } & \text { Shear stress transport } \\ \text { TE } & \text { Trailing edge } \\ \text { TLP } & \text { Tip loaded propeller } \\ \text { TM } & \text { Transition model }\end{array}$

\section{References}

1. Rijpkema, D.; Baltazar, J.; Falcão de Campos, J. Viscous flow simulations of propellers in different Reynolds number regimes. In Proceedings of the Fourth International Symposium on Marine Propulsors, Austin, TX, USA, 31 May-June 42015. 
2. Sánchez-Caja, A.; González-Adalid, J.; Pérez-Sobrino, M.; Sipilä, T. Scale effects on tip loaded propeller performance using a RANSE solver. Ocean Eng. 2014, 88, 607-617. [CrossRef]

3. Dong, X.Q.; Li, W.; Yang, C.J.; Noblesse, F. RANSE-based Simulation and Analysis of Scale Effects on Open-Water Performance of the PPTC-II Benchmark Propeller. J. Ocean Eng. Sci. 2018, 3, 186-204. [CrossRef]

4. Müller, S.B.; Abdel-Maksoud, M.; Hilbert, G. Scale effects on propellers for large container vessels. In Proceedings of the First Internatioal Symposium on Marine Propulsors, Trondheim, Norway, 22-24 June 2009.

5. Baltazar, J.; Rijpkema, D.; Falcão de Campos, J. On the use of the $\gamma-R e_{\theta}$ transition model for the prediction of the propeller performance at model-scale. Ocean Eng. 2018, 170, 6-19. [CrossRef]

6. Baltazar, J.; Rijpkema, D.; Falcão de Campos, J. Prediction of the Propeller Performance at Different Reynolds Number Regimes with RANS. In Proceedings of the 6th International Symposium on Marine Propulsors, SMP'19, Rome, Italy, 26-30 May 2019.

7. Amromin, E. Estimations of scale effects on blade cavitation. J. Phys. Conf. Ser. IOP Publ. 2015, 656. [CrossRef]

8. Viitanen, V.; Siikonen, T.; Sánchez-Caja, A. Numerical Viscous Flow Simulations of Cavitating Propeller Flows at Different Reynolds Numbers. In Proceedings of the 6th International Symposium on Marine Propulsors, SMP'19, Rome, Italy, 26-30 May 2019.

9. Miettinen, A.; Siikonen, T. Application of pressure- and density-based methods for different flow speeds. Int. J. Numer. Methods Fluids 2015, 79, 243-267. doi:10.1002/fld.4051. [CrossRef]

10. Viitanen, V.M.; Siikonen, T. Numerical simulation of cavitating marine propeller flows. In Proceedings of the 9th National Conference on Computational Mechanics (MekIT'17), Trondheim, Norway, 11-12 May 2017; International Center for Numerical Methods in Engineering (CIMNE): Trondheim, Norway, 2017; pp. 385-409. ISBN 978-84-947311-1-2.

11. Miettinen, A. Simple Polynomial Fittings for Steam, CFD/THERMO-55-2007; Report 55; Laboratory of Applied Thermodynamics, Aalto University: Espoo, Finland, 2007.

12. Spalart, P.R.; Deck, S.; Shur, M.; Squires, K.; Strelets, M.K.; Travin, A. A new version of detached-eddy simulation, resistant to ambiguous grid densities. Theor. Comput. Fluid Dyn. 2006, 20, 181-195. [CrossRef]

13. Menter, F.R. Two-equation eddy-viscosity turbulence models for engineering applications. AIAA J. 1994, 32, 1598-1605. [CrossRef]

14. Langtry, R.B. A Correlation-Based Transition Model Using Local Variables for Unstructured Parallelized CFD Codes. Ph.D. thesis, University of Stuttgart, Stuttgart, Germany, 2006.

15. Menter, F.; Langtry, R.; Likki, S.; Suzen, Y.; Huang, P.; Völker, S. A correlation-based transition model using local variables Part I: model formulation. J. Turbomach. 2006, 128, 413-422. [CrossRef]

16. Langtry, R.; Menter, F.; Likki, S.; Suzen, Y.; Huang, P.; Völker, S. A correlation-based transition model using local variables Part II: Test cases and industrial applications. J. Turbomach. 2006, 128, 423-434. [CrossRef]

17. Hellsten, A. Curvature corrections for algebraic Reynolds stress modeling: a discussion. AIAA J. 2002, 40,1909-1911. [CrossRef]

18. Hellsten, A.; Wallin, S.; Laine, S. Scrutinizing curvature corrections for algebraic Reynolds stress models. In Proceedings of the 32nd AIAA Fluid Dynamics Conference, AIAA, St. Louis, MO, USA, 24-26 June 2002; AIAA Paper 2002-2963.

19. Menter, F. Influence of freestream values on $k-\omega$ turbulence model predictions. AIAA J. 1992, 30, 1657-1659. [CrossRef]

20. Shur, M.; Spalart, P.; Strelets, M.; Travin, A. Detached-Eddy Simulation of an Airfoil at High Angle of Attack. In Proceedings of the 4th International Symposium on Engineering Turbulence Modelling and Experiments, Ajaccio, Corsica, France, 24-26 May 1999; pp. 669-678.

21. Strelets, M. Detached eddy simulation of massively separated flows. In Proceedings of the 39th AIAA Aerospace Sciences Meeting and Exhibit, Reno, NV, USA, 8-11 January 2001; AIAA Paper 2001-0879.

22. Frikha, S.; Coutier-Delgosha, O.; Astolfi, J.A. Influence of the cavitation model on the simulation of cloud cavitation on 2D foil section. Int. J. Rotat. Mach. 2009, 2008. [CrossRef]

23. Choi, Y.H.; Merkle, C.L. The application of preconditioning in viscous flows. J. Comput. Phys. 1993, 105, $207-230$. [CrossRef]

24. Sipilä, T. RANS Analyses of Cavitating Propeller Flows. Licentiate Thesis, School of Engineering, Aalto University, Espoo, Finland, 2012. 
25. Viitanen, V.M.; Hynninen, A.; Sipilä, T.; Siikonen, T. DDES of Wetted and Cavitating Marine Propeller for CHA Underwater Noise Assessment. J. Marine Sci. Eng. 2018, 6, 56. [CrossRef]

26. Heinke, H.J. Potsdam Propeller Test Case (PPTC). Cavitation Tests with the Model Propeller VP1304; SVA Potsdam Model Basin Report No.3753; Schiffbau-Versuchsanstalt Potsdam GmbH: Potsdam, Germany, 2011.

27. Viitanen, V.; Hynninen, A.; Lübke, L.; Klose, R.; Tanttari, J.; Sipilä, T.; Siikonen, T. CFD and CHA simulation of underwater noise induced by a marine propeller in two-phase flows. In Proceedings of the Fifth International Symposium on Marine Propulsors (smp'17), Espoo, Finland, 12-15 June 2017.

(C) 2020 by the authors. Licensee MDPI, Basel, Switzerland. This article is an open access article distributed under the terms and conditions of the Creative Commons Attribution (CC BY) license (http:/ / creativecommons.org/licenses/by/4.0/). 